\title{
Perspectives on high-intensity interval exercise for health promotion in children and adolescents
}

This article was published in the following Dove Press journal:

Open Access Journal of Sports Medicine

Number of times this article has been viewed

\author{
Bert Bond' \\ Kathryn L Weston ${ }^{2}$ \\ Craig A Williams' \\ Alan R Barker' \\ 'Children's Health and Exercise \\ Research Centre, Sport and Health \\ Sciences, College of Life and \\ Environmental Sciences, University of \\ Exeter, Exeter, UK ${ }^{2}$ School of Health \\ and Social Care, Teesside University, \\ Middlesbrough, UK
}

\begin{abstract}
Physical activity lowers future cardiovascular disease (CVD) risk; however, few children and adolescents achieve the recommended minimum amount of daily activity. Accordingly, there is virtue in identifying the efficacy of small volumes of high-intensity exercise for health benefits in children and adolescents for the primary prevention of CVD risk. The purpose of this narrative review is to provide a novel overview of the available literature concerning high-intensity interval-exercise (HIIE) interventions in children and adolescents. Specifically, the following areas are addressed: 1) outlining the health benefits observed following a single bout of HIIE, 2) reviewing the role of HIIE training in the management of pediatric obesity, and 3) discussing the effectiveness of school-based HIIE training. In total, 39 HIIE intervention studies were included in this review. Based upon the available data, a single bout of high-intensity exercise provides a potent stimulus for favorable, acute changes across a range of cardiometabolic outcomes that are often superior to a comparative bout of moderate-intensity exercise (14 studies reviewed). HIIE also promotes improvements in cardiorespiratory fitness and cardiometabolic health status in overweight and obese children and adolescents (10 studies reviewed) and when delivered in the school setting (15 studies reviewed). We thus conclude that high-intensity exercise is a feasible and potent method of improving a range of cardiometabolic outcomes in children and adolescents. However, further work is needed to optimize the delivery of HIIE interventions in terms of participant enjoyment and acceptability, to include a wider range of health outcomes, and to control for important confounding variables (eg, changes in diet and habitual physical activity). Finally, research into the application of HIIE training interventions to children and adolescents of different ages, sexes, pubertal status, and sociocultural backgrounds is required. Keywords: vigorous physical activity, primary prevention, pediatric, cardiometabolic disease
\end{abstract}

\section{Introduction}

Cardiovascular diseases (CVDs) are the leading cause of global mortality, and CVD risk-factor status in early life is associated with atherosclerotic progression not only in youth ${ }^{1}$ but also in early adulthood. ${ }^{2}$ Therefore, there is a strong rationale for the identification of effectual interventions that can modulate CVD risk factors in early life for the primary prevention of atherosclerosis. One such intervention is the promotion of physical activity, as time spent performing moderate- to vigorous-intensity physical activity in youth is inversely associated with future CVD risk. ${ }^{3}$ However, only a fifth of children and adolescents achieve the recommended ${ }^{4}$ daily minimum of 60 minutes of moderate- to vigorous-intensity physical activity, ${ }^{5}$ and recent metaanalysis has highlighted that school-based physical activity interventions have only a
Children's Health and Exercise Research

Centre, Sport and Health Sciences,

College of Life and Environmental

Sciences, University of Exeter, St Luke's

Campus, Heavitree Road,

Exeter EXI 2LU, UK

Tel +44 I392 722766

Fax +44 I392 724726

Email A.R.Barker@exeter.ac.uk 
small effect ( $\sim 4$ minutes) on increasing overall moderate- to vigorous-intensity activity levels in children ${ }^{6}$ and adolescents. ${ }^{7}$ Therefore, there is an interest in identifying whether smaller volumes of physical activity can be optimized for the health of children and adolescents.

Both cross-sectional $^{8}$ and longitudinal ${ }^{9}$ data have demonstrated that only time spent performing vigorous-intensity (not moderate- or light-intensity) physical activity is favorably related to CVD risk factors in children and adolescents. These findings are striking, not only due to the small amount of daily vigorous-intensity activity required ( 7 and 4 minutes, respectively) but also because time spent performing light-intensity physical activity was positively related to adiposity. ${ }^{9}$ Data from the European Youth Heart Study similarly reported that time spent performing vigorous- but not moderate-intensity activity was associated with lower adiposity in children ${ }^{10}$ and adolescents. ${ }^{11}$ Furthermore, only vigorous-intensity activity is favorably associated with blood-vessel function in children ${ }^{12}$ which is important, as an impairment in endothelial function is the earliest detectable manifestation of atherosclerosis ${ }^{13}$ and a prerequisite for deleterious structural changes to the vessels. ${ }^{14}$ Therefore, the promotion of short bouts of vigorous-intensity activity in youth appears to be an important consideration regarding the primary prevention of CVD.

Several review articles have recently been published regarding the role high-intensity interval exercise (HIIE) training might play in the promotion of cardiometabolic health in children and adolescents. ${ }^{15-18}$ However, these reviews failed to incorporate fully what we know from acute HIIE studies into their conclusions, which are conceptually important for some health outcomes, and often exclude training studies of $<4$ weeks in duration. ${ }^{15,16}$ Additionally, there is a lack of distinction regarding the effects of HIIE training between different pediatric groups (eg, overweight/obese groups), and the potential challenges facing researchers when conducting HIIE training interventions in settings beyond the laboratory have yet to be addressed. Finally, there is a wide interpretation of what does and does not constitute HIIE in these review papers, with one failing to define this term ${ }^{17}$ and others including only studies of intensity $\geq 64 \%$ peak oxygen uptake $\left(\mathrm{VO}_{2 \text { peak }}\right)$, $\geq 85 \%$ peak heart rate $\left(\mathrm{HR}_{\max }\right),{ }^{15}$ and $\geq 90 \% \mathrm{VO}_{2 \text { peak }}{ }^{18}$ The purpose of this narrative review is to address these limitations and provide a contemporary perspective on the potential for high-intensity exercise to promote cardiometabolic health in children and adolescents. As such, we have defined highintensity exercise as an exercise stimulus $\geq 70 \% \mathrm{VO}_{2 \text { peak }}$ (or equivalent, such as $\mathrm{HR}_{\max }$ ), as this likely represents an intensity greater than the lactate threshold, ${ }^{19}$ which demarcates the upper boundary of the moderate-intensity domain and includes both constant and interval protocols. Specifically, this review outlines the role HIIE may play in health promotion by 1) outlining the health benefits observed following a single bout of HIIE, 2) reviewing the role of HIIE training in the management of pediatric obesity, and 3) discussing the effectiveness of school-based HIIE training.

\section{Acute high-intensity exercise and health outcomes}

The chronic benefits of habitual physical activity are thought to be related to repeated, acute responses to a single exercise bout. ${ }^{20,21}$ For example, the cardiometabolic changes typically observed after a bout of exercise are transient, but these changes can be experienced on a routine basis following regular exercise. Daily exercise thus reduces total exposure to a less favorable cardiometabolic profile over the life span, and in this manner reduces the risk of chronic disease. In line with this concept, it has repeatedly been demonstrated that those who are physically active are characterized by a lower lipemic response to a high-fat meal; however, this benefit is lost following 48 hours of exercise abstention. ${ }^{22,23}$ Additionally, Freese et $\mathrm{al}^{21}$ demonstrated that the magnitude of reduction in postprandial lipemia after a single bout of sprint interval cycling was comparable to that observed after 6 weeks of sprint interval training, suggesting that habitual physical activity and exercise training do not confer favorable metabolic changes beyond a "last bout" effect. Therefore, there is necessity in examining the acute responses to a single bout of HIIE, and (where possible) in comparison with a bout of more traditional continuous aerobic endurance exercise (AEE) training. This perspective is currently missing from existing review papers in this field, ${ }^{15-18}$ which we address here. The available acute high-intensity exercise studies in children and adolescents are summarized in Table 1, and include high-intensity running and cycling exercise bouts and repeated sprint-cycling intervals. ${ }^{24}$ Of the 14 studies in Table 1, 10 included either a high-fat or high-glucose test meal in order to observe how a bout of highintensity exercise may protect against a metabolic "challenge", while 4 studies directly quantified vascular function. Only one study compared whether responses to HIIE differed in normalweight compared to obese children. ${ }^{25}$

\section{Postprandial lipemia}

Most of the day may be spent in the postprandial state, and elevations in nonfasting plasma triacylglycerol (TAG) concentration during adolescence are positively related to CVD events in later life. ${ }^{26}$ Four $^{24,27-29}$ of the HIIE and postprandial 


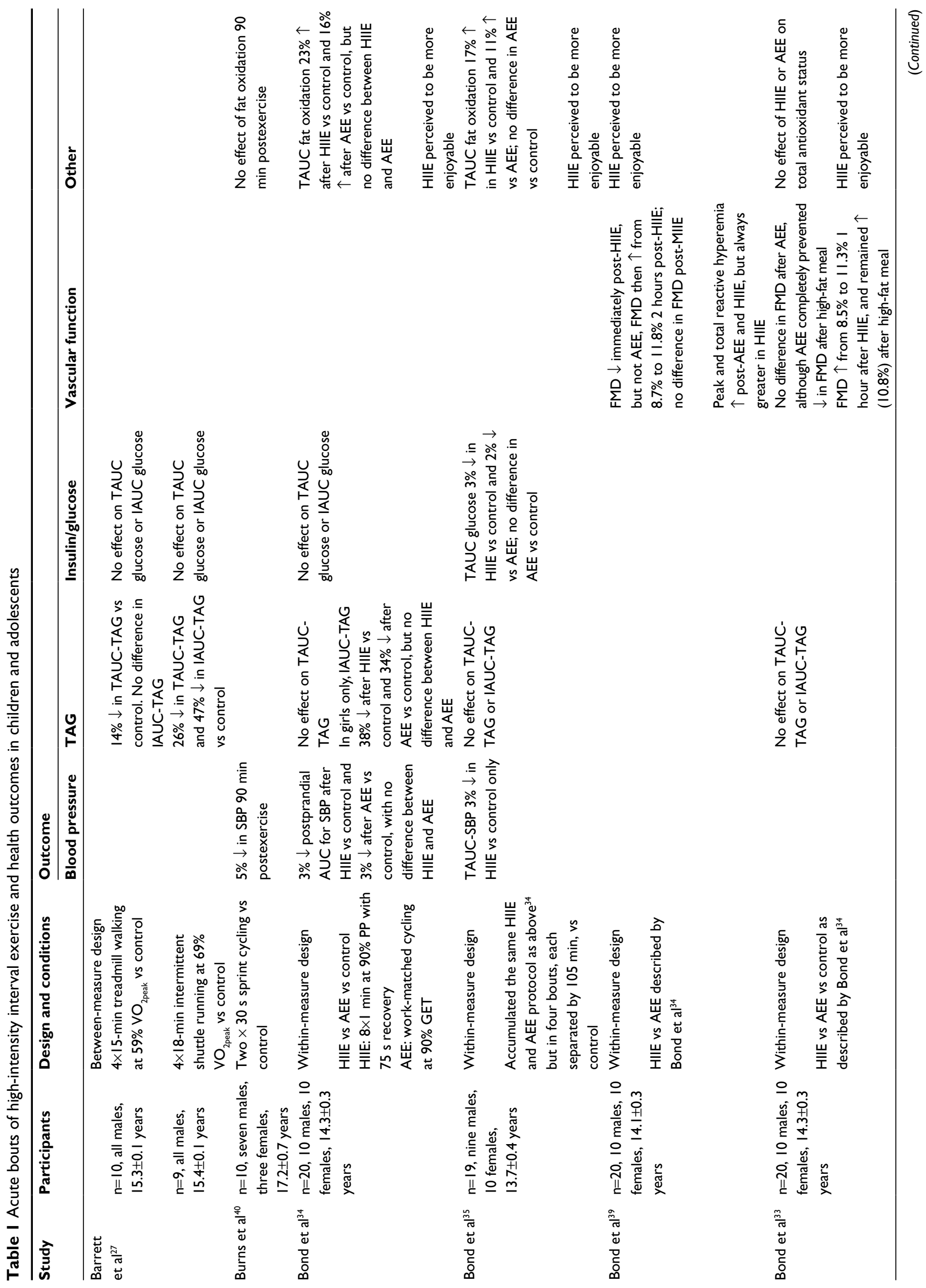




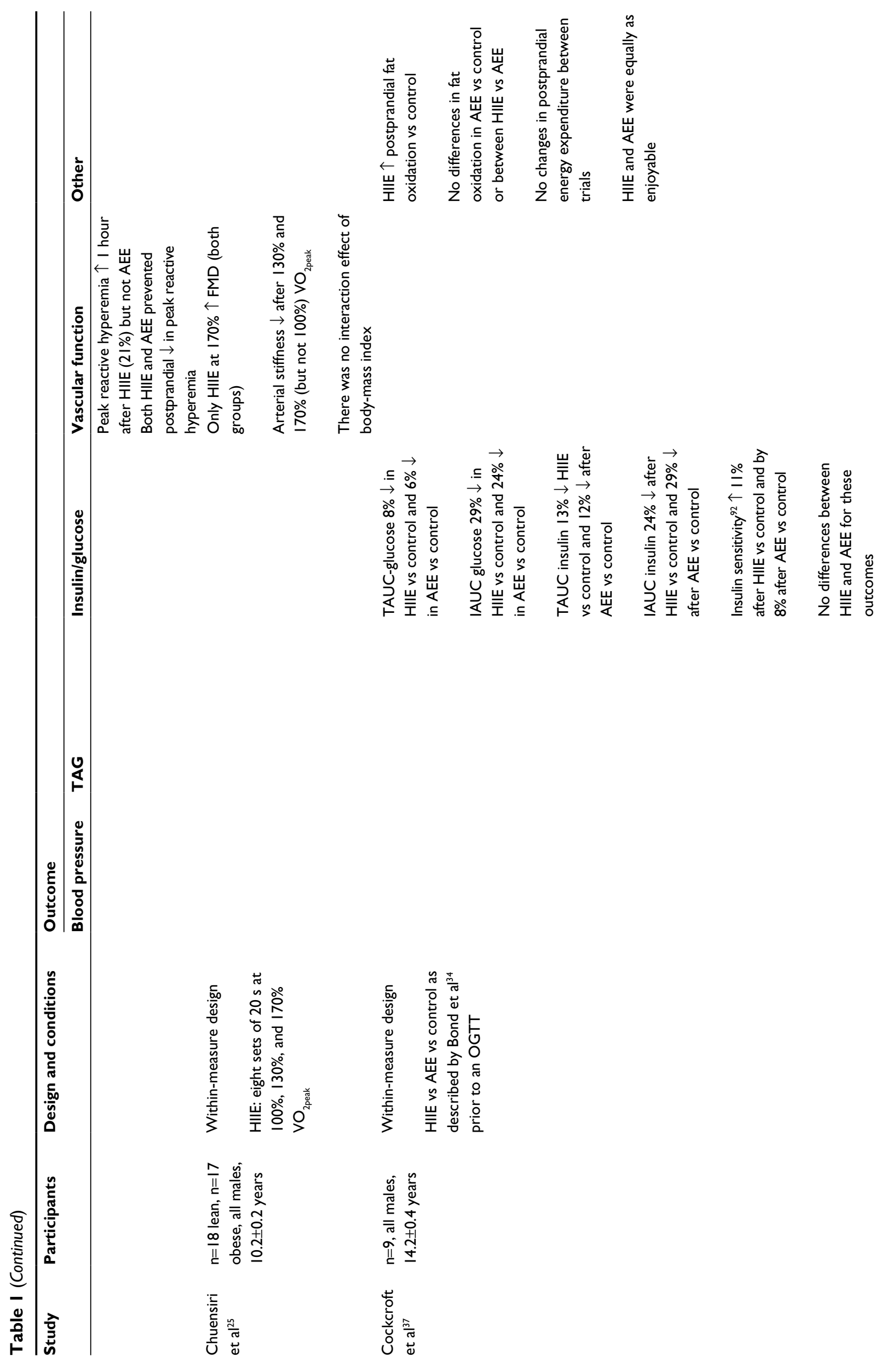




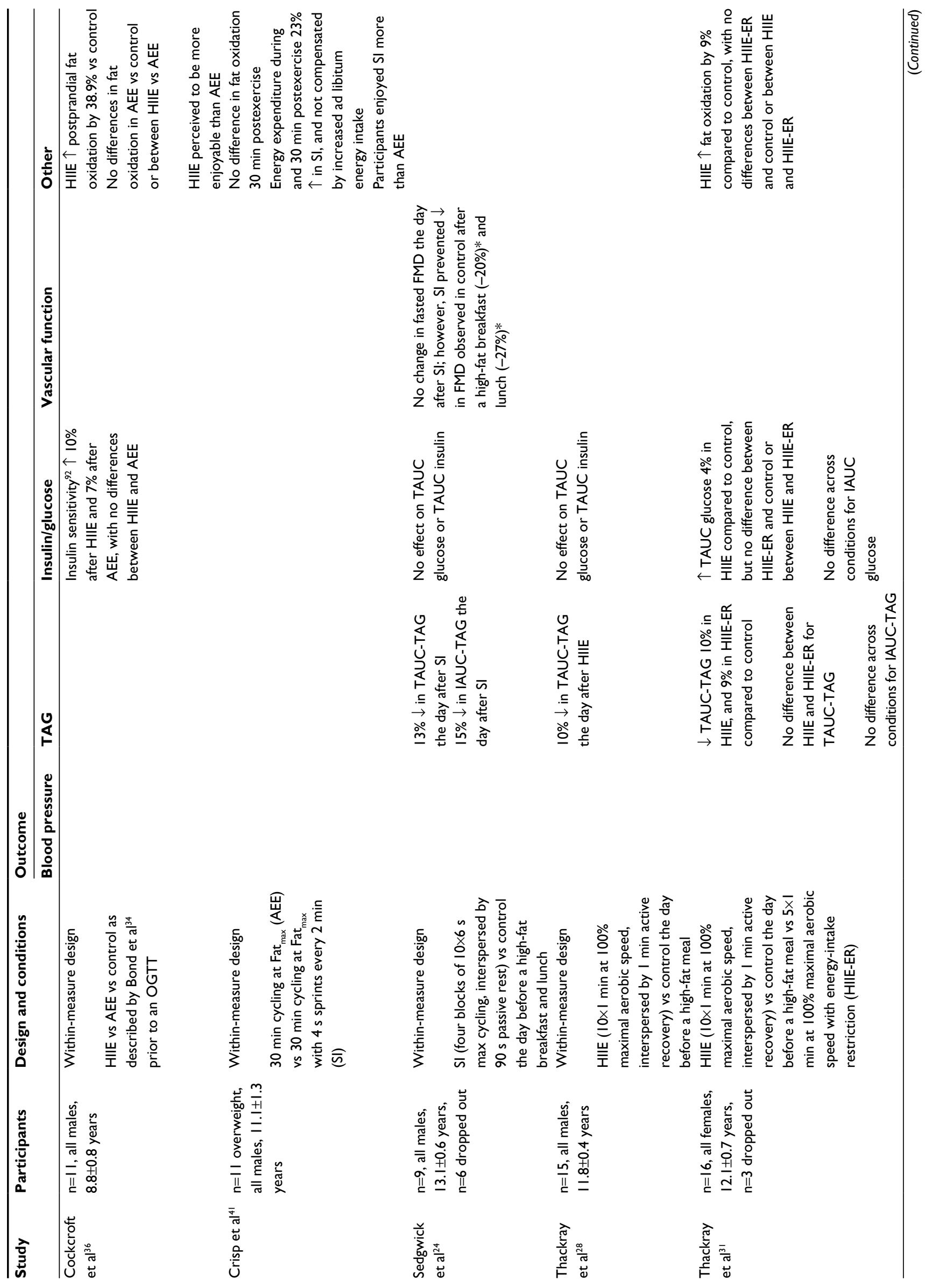



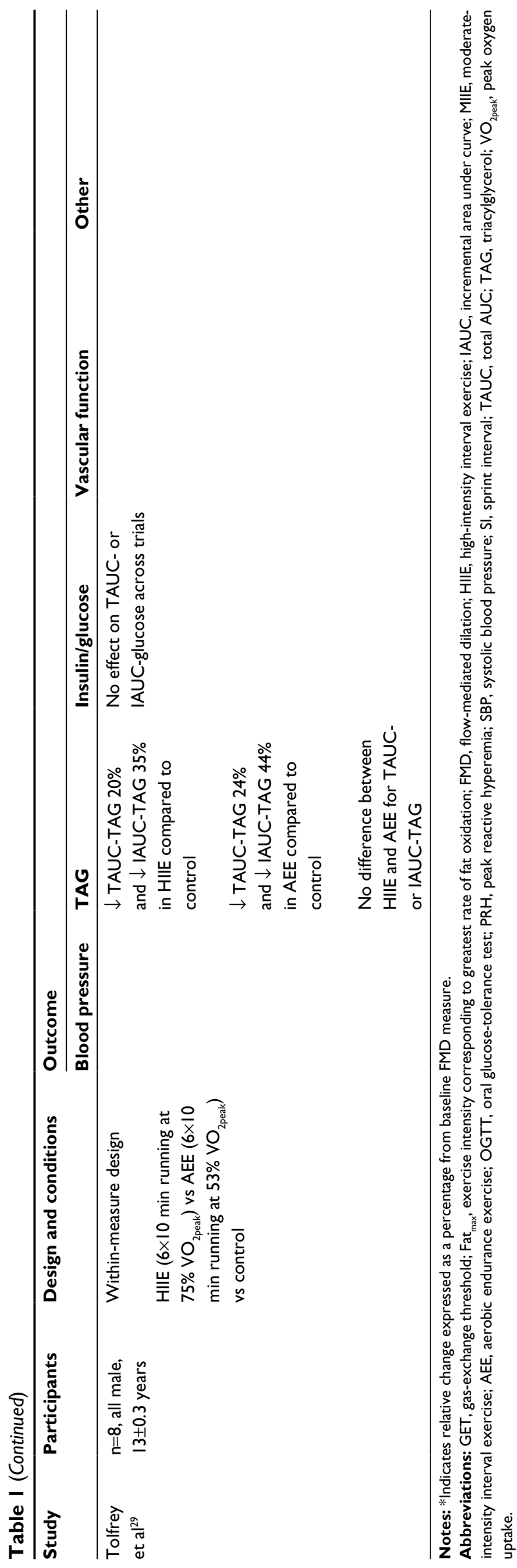

lipemia studies adopted a 2-day protocol, whereby the exercise bout was completed in the afternoon or evening, and the participants consumed a standardized evening meal before being transported to the laboratory the following morning after an overnight fast. One benefit of this design is that it coincides with the delayed peak in lipoprotein-lipase activity postexercise, ${ }^{30}$ which is responsible for the hydrolysis of TAG-rich lipoproteins and mechanistically linked to attenuation of postprandial lipemia following exercise. All of these investigations demonstrated significant reductions in the total area under the curve (TAUC) for TAG of $10 \%-26 \%$, and all but one ${ }^{31}$ demonstrated a $15 \%-47 \%$ reduction in incremental AUC (IAUC) for TAG. These data are in line with the typical attenuation in postprandial lipemia following a bout of AEE in pediatric groups. ${ }^{32}$ Only one of these studies contrasted HIIE to AEE, ${ }^{29}$ and reported a similar reduction in postprandial lipemia following 60 minutes ( $6 \times 10$-minute bouts) of treadmill running at $75 \% \mathrm{VO}_{2 \text { peak }}$ and $53 \% \mathrm{VO}_{2 \text { peak }}$ in boys, despite differences in energy expenditure.

Collectively, data indicate that an acute bout of HIIE has the potential for attenuating postprandial lipemia in young people, although these benefits are likely to be observed the day after the exercise bout, ${ }^{33-35}$ thereby implicating a key role for the delayed increase in lipoprotein-lipase activity. Currently, the large differences in the HIIE dose, timing and composition of test meals, and lack of mechanistic insight mean that it is not possible to identify how HIIE may be optimized to modify postprandial lipemia. However, postprandial lipemia is not attenuated post-HIIE in adolescents, despite a $\sim 20 \%$ increase in resting fat oxidation during the postprandial period, ${ }^{34,35}$ demonstrating that these outcomes are unrelated when exercise is performed the same day as the test meal, although this might not be the case the following day. ${ }^{31}$ An increase in postprandial 3-hydroxybutyrate after HIIE was associated with lower TAUC-TAG in one adolescent study, ${ }^{33}$ suggesting that intensity-dependent changes in hepatic very-low-density lipoprotein output might play some as-yet-unexplored role in this group. Additionally, in one study, ${ }^{34}$ IAUC-TAG was lowered after HIIE (and AEE) in girls only, which suggests a sexual dimorphism in exercise response. However, no other data are available exploring the potential effect of sex on postprandial lipemia after exercise in youth. Similarly, no study has identified whether age, pubertal status, or adiposity influences the effect of exercise or exercise intensity.

\section{Glucose and insulin}

The data presented in Table 1 demonstrate favorable effects of a single bout of HIIE on glycemic control and insulin 
sensitivity following the consumption of an oral glucosetolerance test in children ${ }^{36}$ and adolescents, ${ }^{37}$ although the magnitude of change was greater in the former, suggesting an influence of age and/or biological maturation. In these studies, 23 minutes of HIIE was as effectual as a work-matched bout ( $\sim 30$ minutes) of AEE. Interestingly, this same HIIE stimulus may provide small improvements in glycemic control following a high-fat meal in adolescent boys and girls, but only when accumulated over the course of the day. ${ }^{34,35}$ No pediatric study has yet identified whether HIIE accumulated in several bouts is as effective for cardiometabolic health outcomes, and this remains an interesting avenue for future research, given that young people rarely perform exercise for longer than 10 minutes at a time. ${ }^{38}$

\section{Vascular function}

Recent pediatric studies have extended this area by including flow-mediated dilation (FMD) as a noninvasive measure of endothelial function, in order to observe how an acute bout of HIIE influences the vasculature directly. It has been demonstrated that $\sim 4$ minutes of supramaximal interval cycling $\left(170 \% \mathrm{VO}_{2 \text { peak }}\right)$ acutely improved FMD in both normal-weight and obese prepubescent boys and lowered arterial stiffness in the obese group to the extent that there were no longer differences between cohorts. ${ }^{25} \mathrm{~A}$ limitation of this early work is that the time course of changes in endothelial function after the exercise bouts was not observed. However, a single bout of HIIE has since been demonstrated to impair endothelial function immediately, but significantly augment FMD 1 and 2 hours later in adolescent boys and girls. ${ }^{39}$ Additionally, this study demonstrated that a comparative (work-matched) bout of AEE cycling did not influence FMD, thereby isolating an independent effect of exercise intensity. In a separate study, adolescent boys and girls consumed a high-fat meal 1 hour after this same HIIE and AEE stimulus. ${ }^{33}$ Again, endothelial function improved 1 hour after HIIE only and remained elevated throughout the postprandial period, despite no changes in postprandial lipemia. These findings indicate that a single bout of HIIE is a superior stimulus for acute improvements in vascular function, even in the absence of any reductions in TAG. Interestingly, while AEE failed to increase FMD acutely in this study, it provided complete protection from the fall in postprandial FMD observed in the nonexercise control trial. Finally, in addition to the favorable acute effect of HIIE on endothelial function, other data are available indicating that HIIE can improve postprandial microvascular function ${ }^{33,39}$ and lower blood pressure (BP), ${ }^{34,35,40}$ indicating a wide range of acute CV benefits.

\section{Perspectives}

The existing evidence indicates that an acute bout of HIIE offers improvements across a range of health outcomes in children and adolescents. Furthermore, the magnitude of these benefits is either similar to $\mathrm{o}^{29,34,36,37,41}$ or superior than $^{33-35,39,42}$ a bout of AEE. Accordingly, there is sufficient premise to explore this field further and provide the basic information on how to optimize the HIIE stimulus when using an acute-exercise model. For example, we do not yet know the dose-response relationship behind the acute HIIE stimulus and subsequent physiological changes, which would help to identify both an "optimal" and "minimal" HIIE dose and how this may differ based on the age, sex, and pubertal status of the participant. It would also be insightful to compare different exercise modalities directly and to identify how manipulating the HIIE stimulus in these ways can preserve or improve participant enjoyment in different groups. Additionally, comparing the accumulation of HIIE over the day to a single HIIE bout remains an unexplored area, but is conceptually important, as children accumulate their habitual physical activity in short bouts throughout the day.

\section{High-intensity interval exercise training and management of pediatric obesity}

The prevalence of childhood obesity has increased in recent years in both developed and developing countries, and represents a major public health challenge. ${ }^{43}$ Obese children are characterized by elevated CVD risk, ${ }^{44}$ reduced cardiorespiratory fitness (CRF), ${ }^{45}$ and an increased risk of future health-related morbidity and premature mortality. ${ }^{46}$ Although multicomponent lifestyle interventions are the cornerstone of managing pediatric obesity, increasing levels of physical activity through exercise represent a fundamental component of most interventions. ${ }^{47}$ Traditional exercise recommendations for managing pediatric obesity typically focus on the completion of continuous AEE ( $<60$ minutes per day, two to four times/week). ${ }^{48}$ Recently, there has been an interest in the potential for time-efficient HIIE training to improve health outcomes in overweight and obese children and adolescents. An overview of the studies $(n=10)$ that have examined the efficacy of HIIE training for improving health outcomes in overweight and obese children and adolescents in comparison with a control or exercise group is given in Table 2. In addition, changes in health outcomes (body size and composition, blood markers, BP, and CRF) following HIIE training are presented in Table 3. 


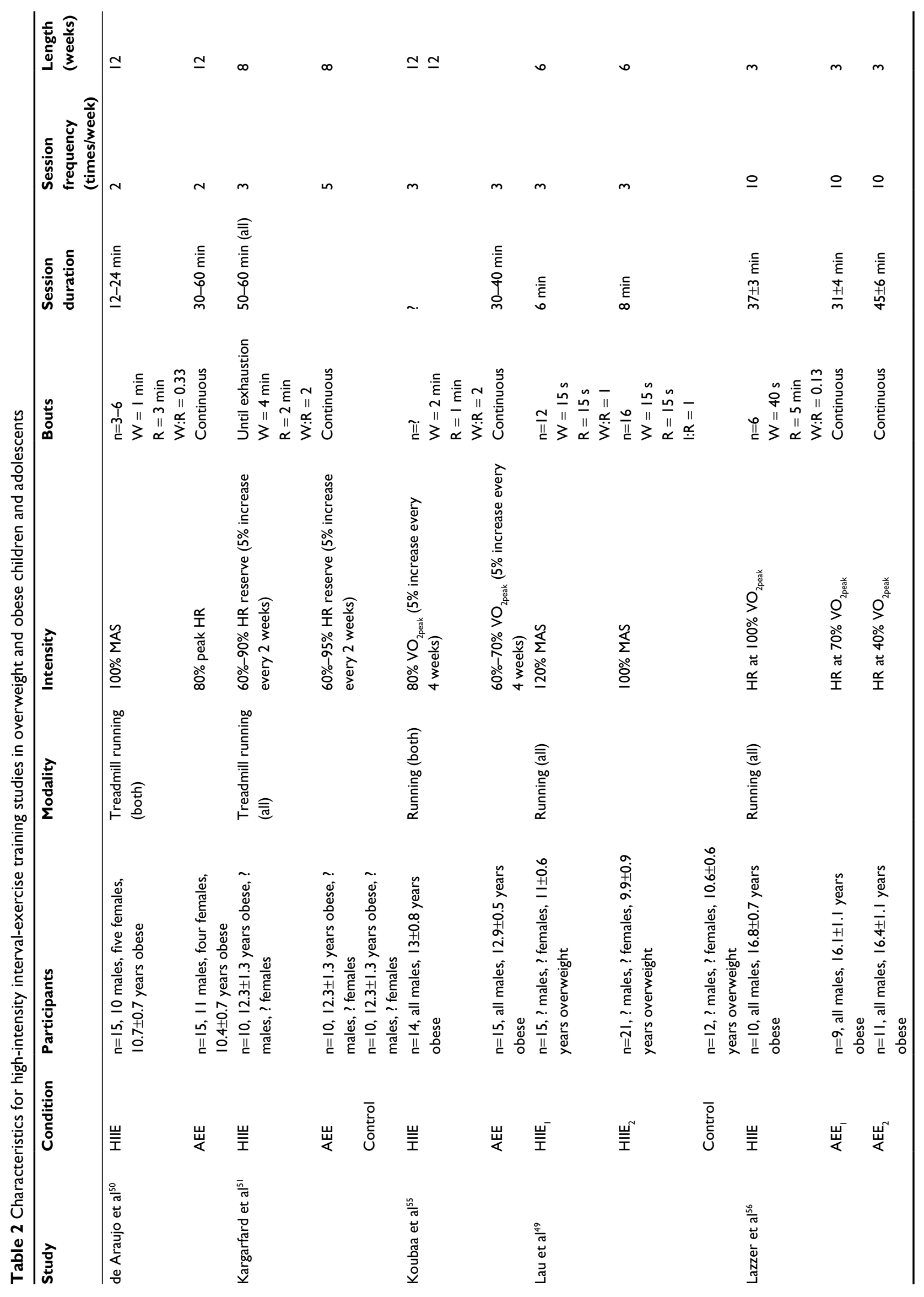




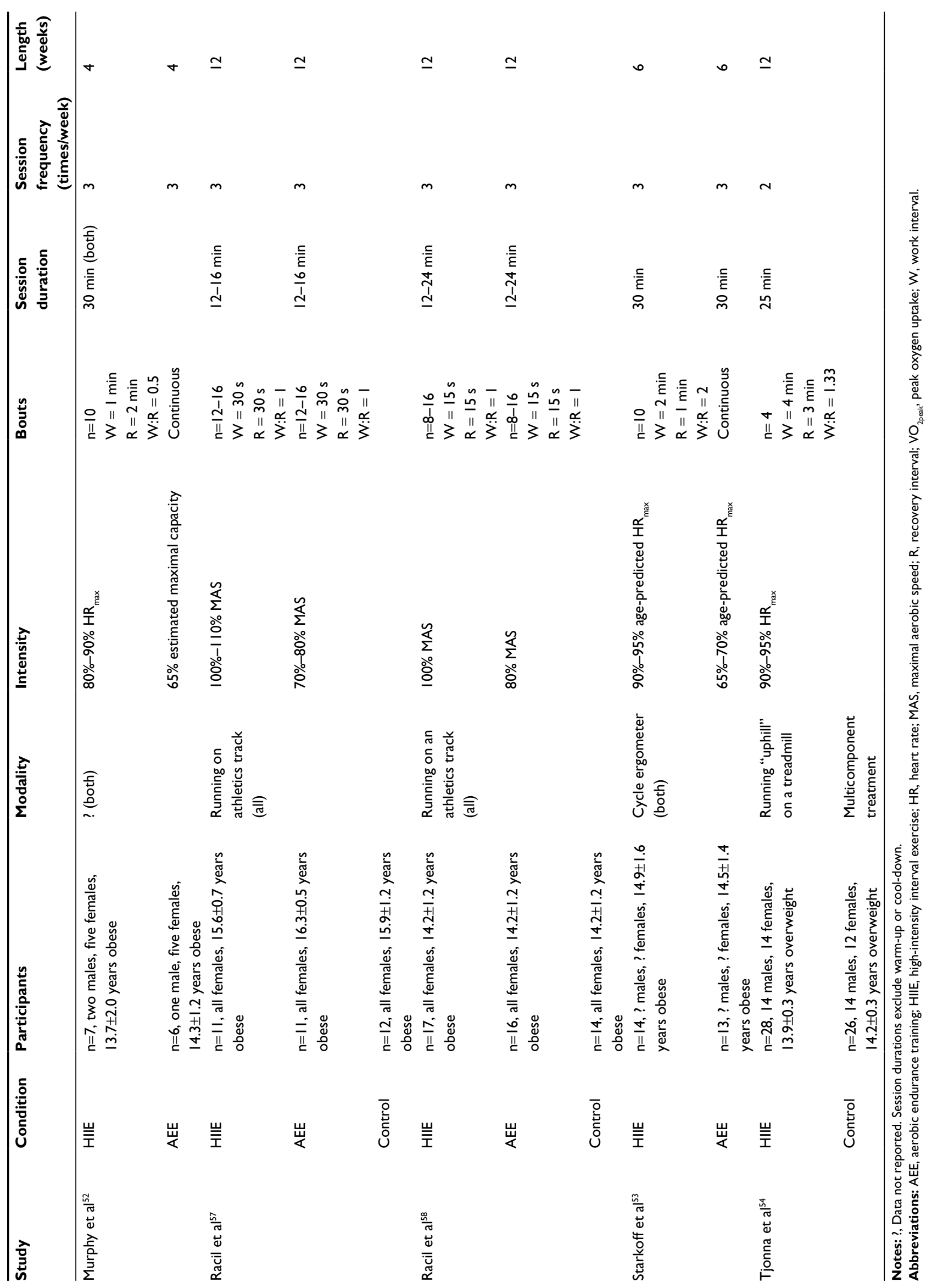




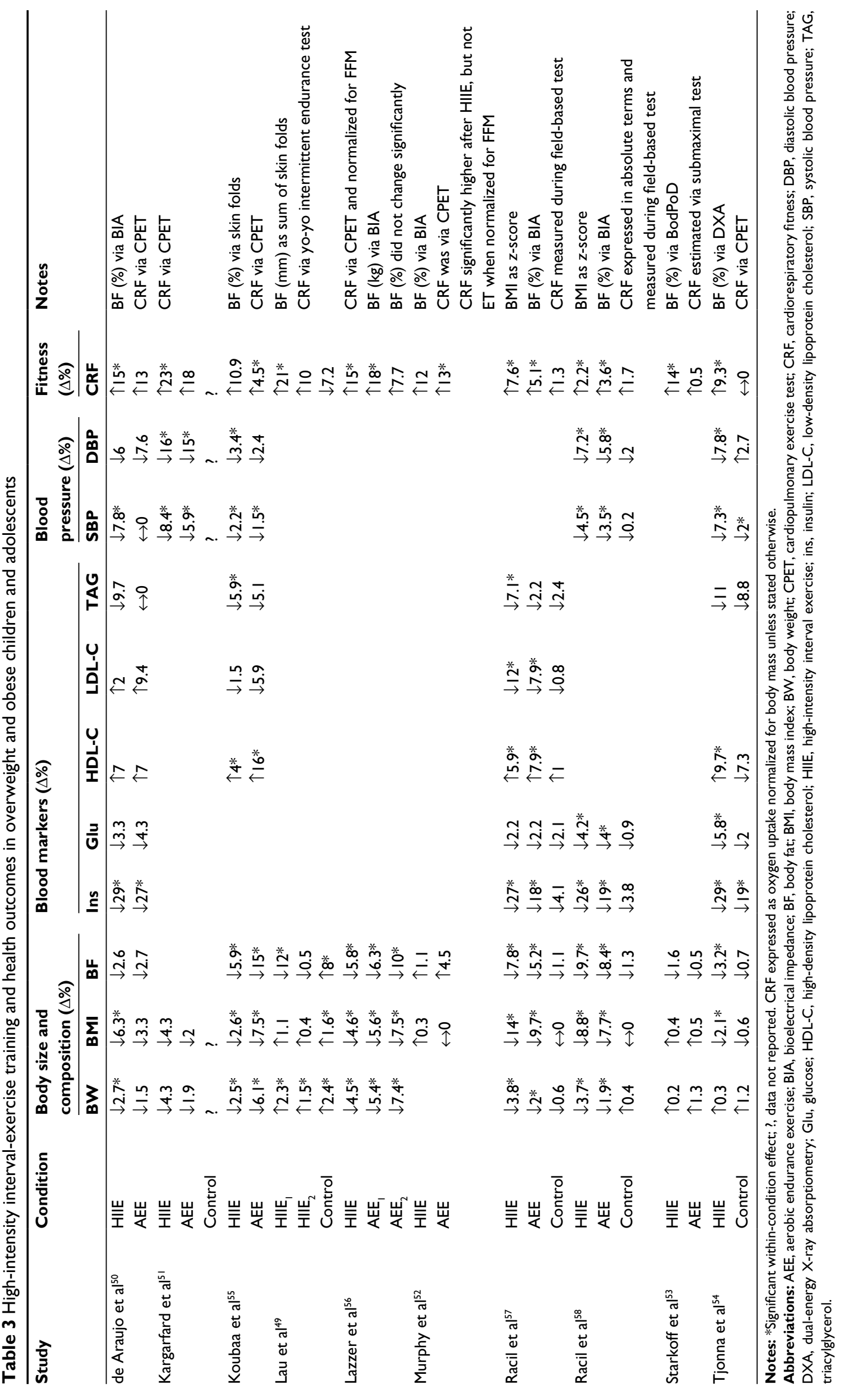




\section{Study characteristics}

Two studies were designed to examine HIIE training in overweight or obese children (age $\sim 10-11$ years), but Lau et al ${ }^{49}$ did not report the pubertal status of the participants, and de Araujo et a ${ }^{50}$ included participants ranging from pre- to midpuberty. The remaining eight studies included an adolescent sample with ages $12.3-16.8$ years. Six studies included both male and female participants in the groups ${ }^{49-54}$ whereas two studies included only male $e^{5,56}$ or female ${ }^{57,58}$ participants. No HIIE training study has examined the interaction of age, pubertal status, and/or sex on health outcomes in overweight or obese children and adolescents.

Of the 10 studies, 5 examined HIIE training in comparison with an AEE training group consisting of $>30$ minutes of continuous submaximal aerobic exercise..$^{50,52,53,55,56}$ Four studies included a control group and a comparative AEE training group consisting of either continuous or interval exercise. ${ }^{49,51,57,58}$ One study had a comparative control group receiving a multicomponent weight management program. ${ }^{54}$ A variety of HIIE training protocols have been adopted, with a duration of 3-12 weeks ${ }^{50,56}$ and a frequency of two to ten sessions per week. ${ }^{50,56}$ The intensity of the work intervals for the HIIE training protocol was typically selected based on a prior incremental test to exhaustion to determine maximal aerobic speed (MAS; 100\%-120\%), ${ }^{49,50,57,58}$ $\mathrm{HR}_{\max }(80 \%-100 \%),{ }^{52,54}$ or $\mathrm{VO}_{2 \text { peak }}(80 \%) .{ }^{55}$ Some studies prescribed the intensity of the AEE work interval based on an estimate of age-predicted $\mathrm{HR}_{\max }(90 \%-95 \%)^{53}$ or HR reserve $(60 \%-90 \%) .{ }^{51}$ The duration of the work intervals ranged from 15 seconds ${ }^{58}$ to 4 minutes,${ }^{54}$ with recovery intervals ranging from 15 seconds $^{58}$ to 3 minutes..$^{50}$ The lowest work:recovery ratio was $0.13^{56}$ and the highest $2 .{ }^{51}$ Excluding a warm-up and cool-down which may take 10-15 minutes, the duration of the HIIE session ranged from $6^{49}$ to 60 minutes.$^{51}$ Koubaa et $a{ }^{55}$ did not report the duration of the HIIE training sessions, as participants completed as many work intervals as possible until exhaustion. Seven studies employed a running modality on either a treadmill $1^{50,51}$ or running track, ${ }^{57,58}$ although three of these did not specify the location of the running. ${ }^{49,55,56}$ One study adopted a cyclingbased HIIE training protocol, ${ }^{53}$ which may be preferable in obese individuals, as it reduces the requirement to "carry" their excess weight and may limit musculoskeletal complications and pain in contrast to running. Murphy et a ${ }^{52}$ did not report the modality for delivering HIIE training. No HIIE training study has formally compared different modalities in overweight or obese children and adolescents.

\section{Body size and composition}

All but one HIIE training study ${ }^{52}$ reported body weight (BW) as an outcome. Six of nine studies reported a decrease in BW following HIIE training, but this was significant in only five studies, with a magnitude ranging from $2.3 \%$ to $4.5 \%{ }^{50,55-58}$ Interestingly, four of these studies employed an HIIE training program lasting 12 weeks in duration, ${ }^{50,55,57,58}$ suggesting longer duration programs may be needed to promote reductions in BW. By contrast, Lazzer et al ${ }^{56}$ observed a significant $4.5 \%$ reduction in $\mathrm{BW}$ following 3 weeks of HIIE training in ten obese adolescent males. The participants performed on average $28 \pm 2$ HIIE training sessions over this time period, compared to a frequency of two to three sessions per week in the 12-week studies ( $\sim 24-36$ sessions), indicating the overall "dose" of HIIE training was similar between the studies. Three studies reported a rise in BW following HIIE training, with two nonsignificant and trivial $(0.2 \%-0.3 \%)^{53,54}$ and one significant for HIIE protocols at $100 \%$ and $120 \%$ MAS (1.5\% and $2.3 \%$, respectively). ${ }^{49}$ The latter study by Lau et $\mathrm{al}^{49}$ also observed a significant increase in BW in controls, with no reported differences between the conditions, suggesting this increase in BW over the intervention was a reflection of growth and maturation.

As overweight and obesity status in children and adolescents is invariably classified using body mass index (BMI) centiles ${ }^{59}$ it is not surprising that all HIIE training studies have reported this outcome. Seven of ten studies reported a reduction in BMI following HIIE training, of which six were significant, with a magnitude of $2.1 \%-14 \% .{ }^{50,54-58}$ The greatest magnitudes of change (8.8\% and $14 \%$ ) in BMI were found by Racil et al, ${ }^{57,58}$ which may be a reflection of reporting BMI using a $z$-score. When BMI is reported in its original units $\left(\mathrm{kg} / \mathrm{m}^{2}\right)$, the magnitude of the reduction ranged from $2.1 \%{ }^{54}$ to $6.3 \%{ }^{50}$ Although Kargarfard et al ${ }^{51}$ reported a $4.3 \%$ reduction in BMI following 8 weeks of HIIE training in obese male and female adolescents, the lack of significance may be attributable to the low sample size $(n=10)$, in contrast to studies showing a significant beneficial effect $(\mathrm{n}=10-28)$. Three studies ${ }^{49,52,53}$ reported a nonsignificant increase $(0.3 \%-1.1 \%)$ in BMI following HIIE training that was similar to other comparativeexercise groups in the respective studies. Of note, however, is that in the study by Lau et al, ${ }^{49}$ the control group experienced a significant $1.6 \%$ increase in BMI, whereas 6 weeks of HIIE training at either $100 \%$ or $120 \%$ MAS did not significantly increase BMI. Therefore, HIIE training may have prevented an increase in BMI, meaning a stagnated BMI response following HIIE training may represent a health benefit. 
Nine of the HIIE training studies documented changes in body fat (BF). Eight of these studies demonstrated a reduction in $\mathrm{BF}$, and this was significant in six studies, with a magnitude of $3.2 \%-12 \%{ }^{49,54-58}$ The large variability in magnitude of reduction in BF may be due to methodological issues, such as the measurement of BF. The largest changes following HIIE training were reported in studies using indirect measures, such as skin-fold thickness $(5.8 \%-12 \%)^{49,55}$ or bioelectrical impedance $(5.8 \%-9.7 \%) .{ }^{56-58}$ When dualenergy X-ray absorptiometry is used, ${ }^{54}$ a lower magnitude of change $(3.2 \%)$ is observed. This shows that changes in BF following HIIE training may be overstated when skin-fold or bioelectrical impedance methodology is used. However, a nonsignificant reduction ${ }^{50}$ or increase $^{52}$ in $\mathrm{BF}$ has been reported using bioelectrical impedance following HIIE training, suggesting other factors may be influential. For example, Tjonna et $\mathrm{al}^{54}$ reported a decrease in total energy, fat, and carbohydrate intake (food diary) and an increase in physical activity (accelerometer) following 12 weeks of HIIE training in 28 overweight adolescents. However, they did not adjust for these confounding factors when analyzing body composition changes. Therefore, it is possible that the beneficial effects on BF following HIIE training may be mediated through changes in diet and/or physical activity.

\section{Blood markers}

Only five HIIE training studies included blood markers of CVD risk as health outcomes, and the types of markers reported varied considerably between studies. Four studies $^{50,54,57,58}$ reported fasting insulin and glucose. All four studies reported a significant reduction in fasted insulin, with a magnitude of $26 \%-29 \% .^{50,54,57,58}$ These four studies were characterized by a 12-week HIIE training protocol with two to three sessions per week, but the work intervals varied markedly from $0.25^{58}$ to 4 minutes $^{54}$ in duration. Despite all four studies showing a reduction in fasting glucose following HIIE training, this was only significant in two studies, which had the largest magnitude of change: $4.2 \%{ }^{58}$ and $5.8 \%{ }^{54}$ Using homeostasis model assessment, a reduction in insulin resistance $e^{50,57,58}$ or an increase in insulin sensitivity ${ }^{54}$ has been observed after HIIE training. In addition, Tjonna et $\mathrm{al}^{54}$ reported beneficial changes to glucose and insulin 2 hours after an oral glucose-tolerance test following HIIE training. Collectively, these findings indicate beneficial changes to fasting and postprandial insulin and glucose health outcomes following HIIE training in obese pediatric groups, but it is unclear if these findings are a chronic adaptation to HIIE or a reflection of the last bout of HIIE performed, as the studies did not specify the timing of the measurements following the final HIIE training session.

Blood lipid outcomes following HIIE training were reported in four studies, ${ }^{50,54,55,57}$ with the exception of LDL cholesterol (LDL-C), which was reported in three studies. ${ }^{50,55,57}$ High-DL cholesterol (HDL-C) increased in all four studies (4\%-9.7\%), with three of these deemed statistically significant. ${ }^{54,55,57}$ For LDL-C, one study showed a statistically significant decrease of $12 \%,{ }^{57}$ while others showed a nonsignificant decrease of $1.5 \%{ }^{55}$ and a nonsignificant increase of $2 \% .^{50}$ Lastly, all four studies showed a reduction in fasting TAG following HIIE training (5.9\%-11\%), but only two of these were deemed statistically significant. ${ }^{55,57}$

\section{Blood pressure}

Despite BP's relative ease in measurement and its association with pediatric obesity, ${ }^{44}$ it is surprising that only five HIIE training studies ${ }^{50,51,54,55,58}$ have included it as a health outcome. All five studies reported a significant decrease in systolic BP (SBP), with a magnitude ranging from $2.2 \%{ }^{55}$ to $8.4 \% .{ }^{51}$ Likewise, all five studies reported a decline in DBP with a magnitude of $3.4 \%-16 \%$, but this was significant in four studies. ${ }^{51,54,55,58}$ Interestingly, all HIIE training studies that reported $\mathrm{BP}$ as an outcome had a program length of 8-12 weeks and incorporated two to three sessions per week. However, the duration of the work intervals across these studies ranged from $0.25^{58}$ to 4 minutes, ${ }^{54}$ suggesting beneficial changes in BP can be achieved using a range of HIIE training protocols.

\section{Cardiorespiratory fitness}

$\mathrm{CRF}$ is independently related with CVD risk factors in children and adolescents, even after controlling for physical activity and $\mathrm{BF}^{60}$ In addition, high $\mathrm{CRF}$ may protect against the development of elevated CVD risk in youth who are obese ("fat-fit" concept), ${ }^{61}$ and high CRF in late adolescence has been associated with a lower risk of premature mortality ${ }^{62}$ in adulthood. Therefore, CRF status in children or adolescents is a powerful marker of current and future health status.

All 10 studies included in this review reported CRF following HIIE training. Six and two studies, respectively, measured CRF objectively using gas analysis (eg, $\mathrm{VO}_{2 \text { peak }}$ ) during a laboratory-based cardiopulmonary exercise test $(\mathrm{CPET})^{50-52,54-56}$ or in a field setting. ${ }^{57,58}$ One study estimated CRF using submaximal HR, ${ }^{53}$ and one study estimated CRF based on performance during the yo-yo intermittent endurance test. ${ }^{49}$ All 10 studies reported an increase in CRF following HIIE training, and this reached significance in eight 
studies, with a magnitude of $2.2 \%-23 \% .{ }^{49-51,53,54,56-58}$ Kargarfard et $\mathrm{al}^{51}$ found the greatest change in CRF (23\%) using an 8-week HIIE training protocol with repeated 4-minute work intervals until exhaustion, which may be explained in part by a very low baseline CRF $\left(\sim 20 \mathrm{~mL} \cdot \mathrm{kg}^{-1} \cdot \mathrm{min}^{-1}\right)$. Racil et al ${ }^{58}$ reported a much lower but significant $2.2 \%$ increase in CRF following 12 weeks of HIIE training. This lower magnitude of change likely reflects the expression of $\mathrm{VO}_{2 \text { peak }}$ in absolute terms $\left(\mathrm{L} \cdot \mathrm{min}^{-1}\right)$, as mean data in the manuscript revealed that $\mathrm{VO}_{2 \text { peak }}$ normalized for BM increased by $\sim 6.5 \%$. The two studies that did not reach significance both measured CRF via a CPET and had a magnitude of change of $10 \%-12 \% .^{52,55}$ Although not significant, it could be argued the magnitude of change in these studies is important, as exercise training programs in health children typically report a 5\%-10\% improvement. ${ }^{63}$ Furthermore, although not significant when normalized for BM, there was a significant $10 \%$ increase in CRF when normalized for fat-free mass. ${ }^{52}$

\section{Perspectives}

Only a small number of studies have examined the effect of HIIE training on health outcomes in obese children and adolescents, and a large proportion of these should be considered pilot data, rather than well-controlled randomized trials. Despite the paucity of available studies, the evidence to date indicates that beneficial changes in body size and composition, blood metabolic markers, BP, and CRF are achievable following HIIE training. In particular, positive improvements in $\mathrm{CRF}$, fasted insulin, and BP were observed in the majority of studies, which in some cases occurred in the absence of changes in body size and composition. This lack of beneficial change in body size and composition in some studies may be due to the short duration of the HIIE training studies conducted to date $\left(<12\right.$ weeks). Tjonna et $\mathrm{al}^{54}$ did include 12-month follow-up data, showing beneficial changes in body composition (and other CVD health outcomes) persisted over time; however, participants were not formally involved in the HIIE training program over this 12-month follow-up period. Future research should consider examining the impact of longer HIIE training programs on health outcomes in obese children and adolescents. Furthermore, the measurement of and statistical adjustment for confounding variables, such as diet and physical activity, is essential for future work to isolate the effect of HIIE training.

Unfortunately, the current review cannot provide specific recommendations regarding potential moderators of HIIE training on health outcomes in obese children and adolescents. Therefore, it is unclear how such factors as age, sex, and biological maturation may interact with health-related adaptations to HIIE training. The current review is also unable to recommend an optimal prescription of HIIE training, largely because of the varied nature of protocols used in the literature. Only one study has formally included two types of HIIE training protocols (100\% vs $120 \%$ MAS) on overweight adolescents after 6 weeks of training. ${ }^{49}$ Using a within-condition analysis, the authors found beneficial effects for BF and CRF only following HIIE training at $120 \%$ MAS, but this was only significant between conditions for BF. Future studies should formally compare different HIIE training protocols in order to optimize the dose-response relationship for different health outcomes. A recent metaanalysis $^{16}$ suggested that the length of the HIIE training program may be important for determining changes in CRF in overweight or obese youth, although it should be noted that the largest $(>20 \%$ ) changes in CRF were found in studies with lengths of $6^{49}$ and $8^{51}$ weeks. Finally, no attempt was made in the current review to contrast the beneficial effects of performing HIIE training compared to the comparisonexercise and/or control group. A meta-analysis comparing HIIE training to comparative exercise groups identified a beneficial effect for HIIE training on CRF (medium effect size) and SBP (small effect size), but not for body composition or cardiometabolic outcomes. ${ }^{16}$ Therefore, although the present review indicates HIIE training may have a beneficial effect on a myriad of CVD risk factors in obese children and adolescents, for most health outcomes the benefits appear similar to performing traditional AEE training that is either continuous or interval in nature.

\section{High-intensity interval exercise training in the school setting}

School-based interventions are often regarded as the most universally applicable and effective way to impact the health of young people. ${ }^{64}$ Nonetheless, schools are busy, often unpredictable places that can create unique challenges and considerations for researchers. Accordingly, this section provides perspectives on some methodological issues pertinent to conducting HIIE training interventions outside the laboratory and gives a concise overview of the effects of school-based HIIE training on cardiometabolic health and fitness.

Table 4 summarizes the characteristics of 15 studies wherein HIIE training intervention took place within schools, HIIE training intensity was quantified, and either CRF or traditional CVD risk factors (eg, body composition, BP, HDL-C, TAG and glucose) assessed. ${ }^{65}$ Thirteen were conducted in European schools ${ }^{66-78}$ and two in Australasia. ${ }^{79,80}$ 


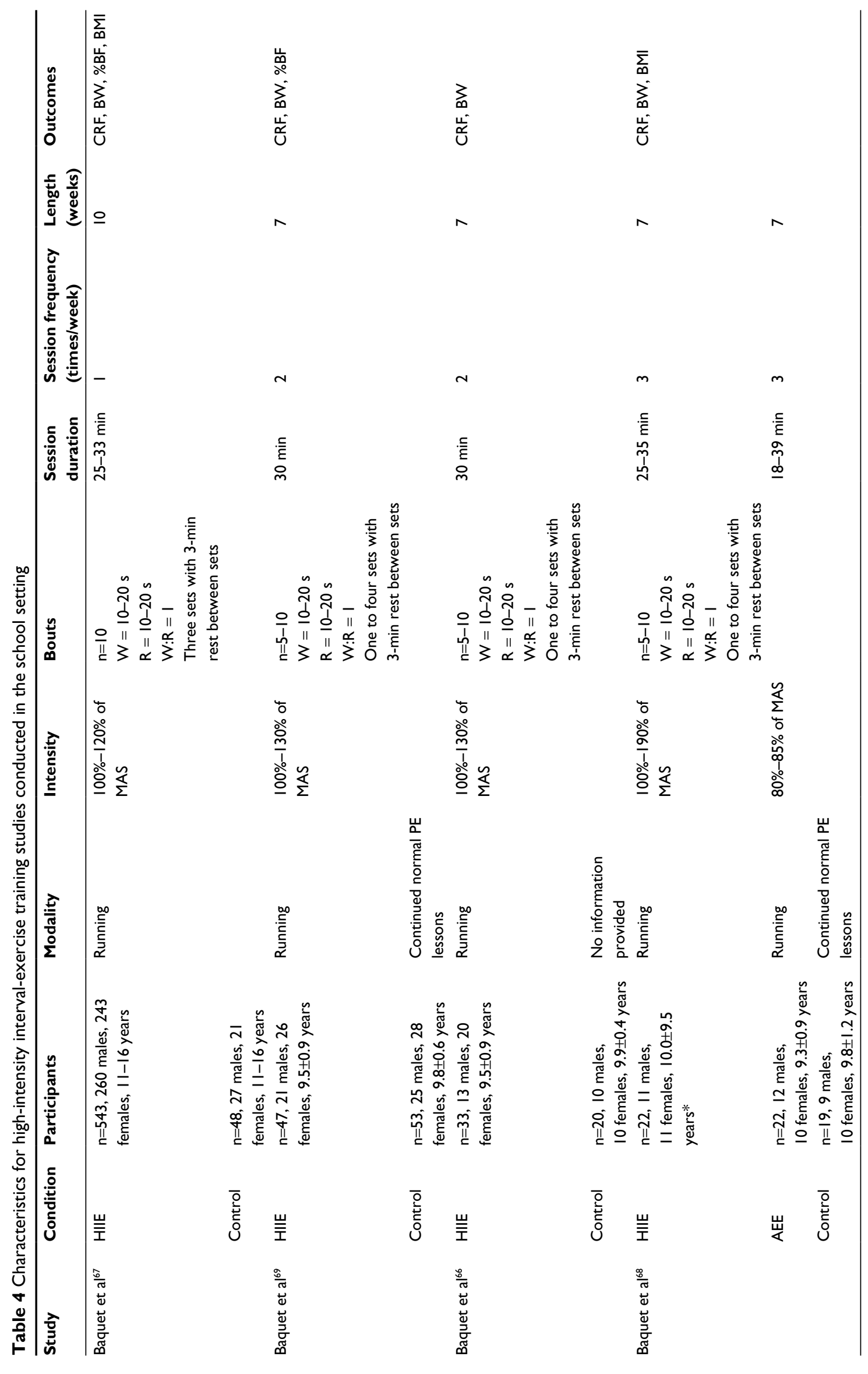




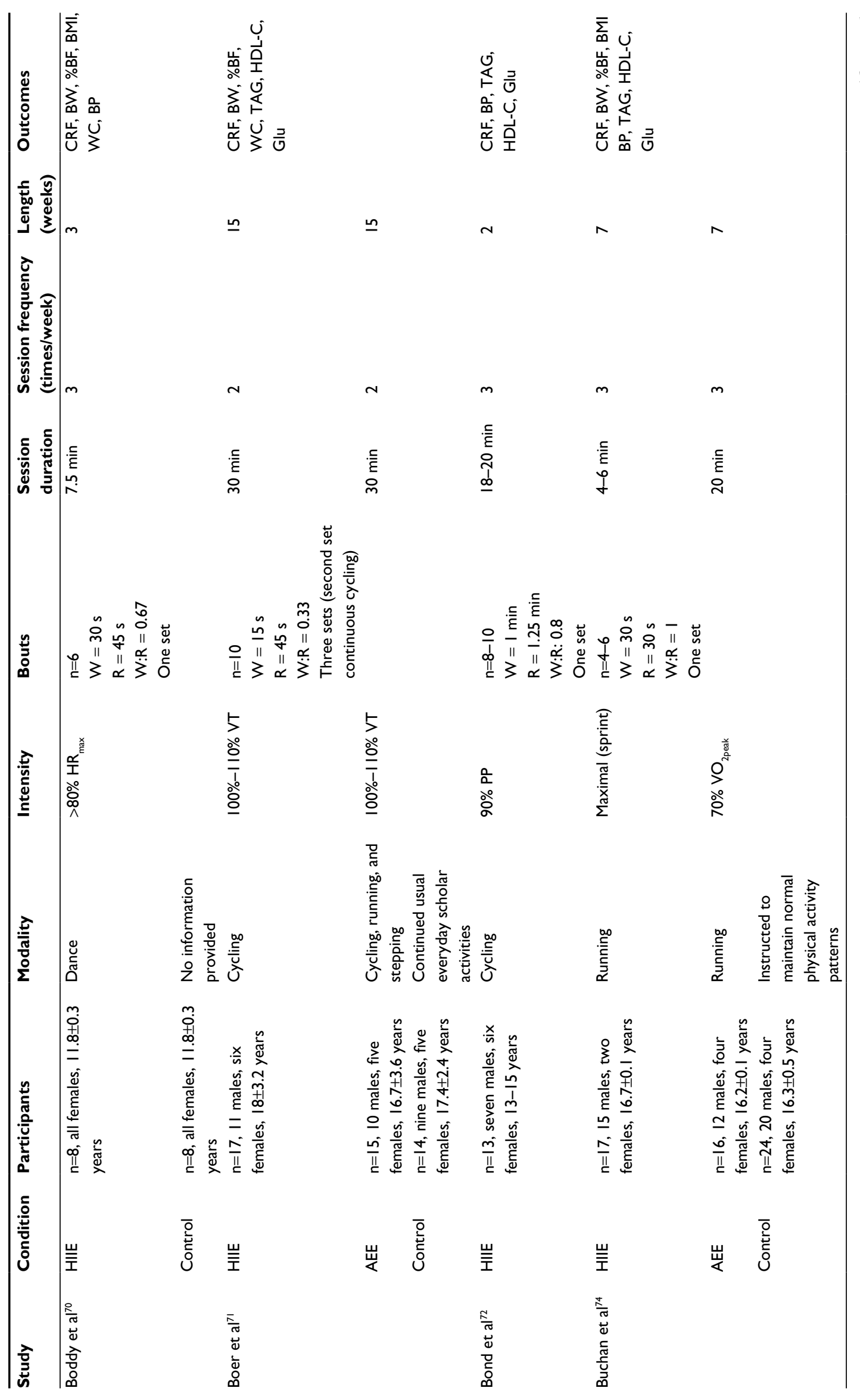




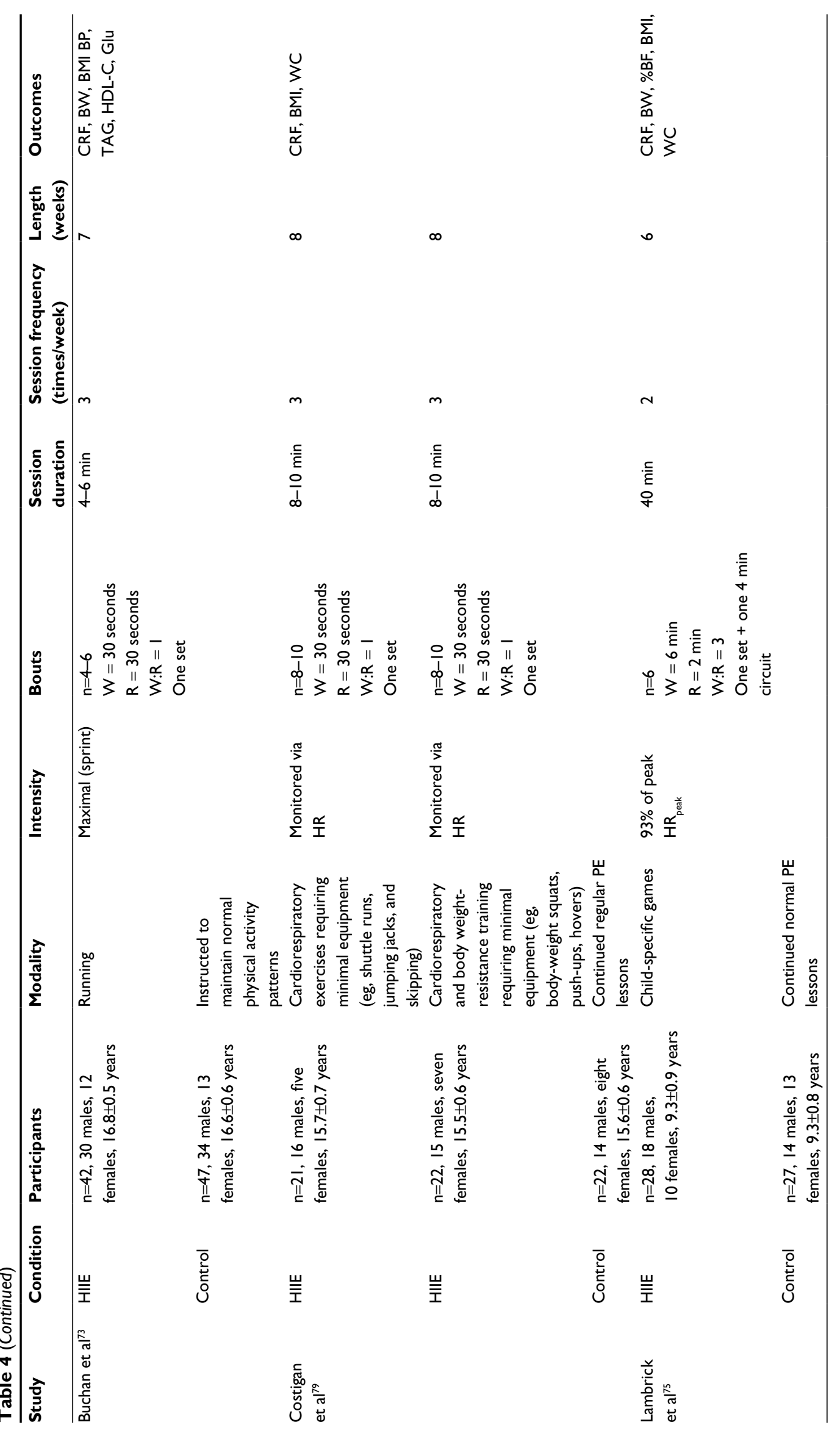




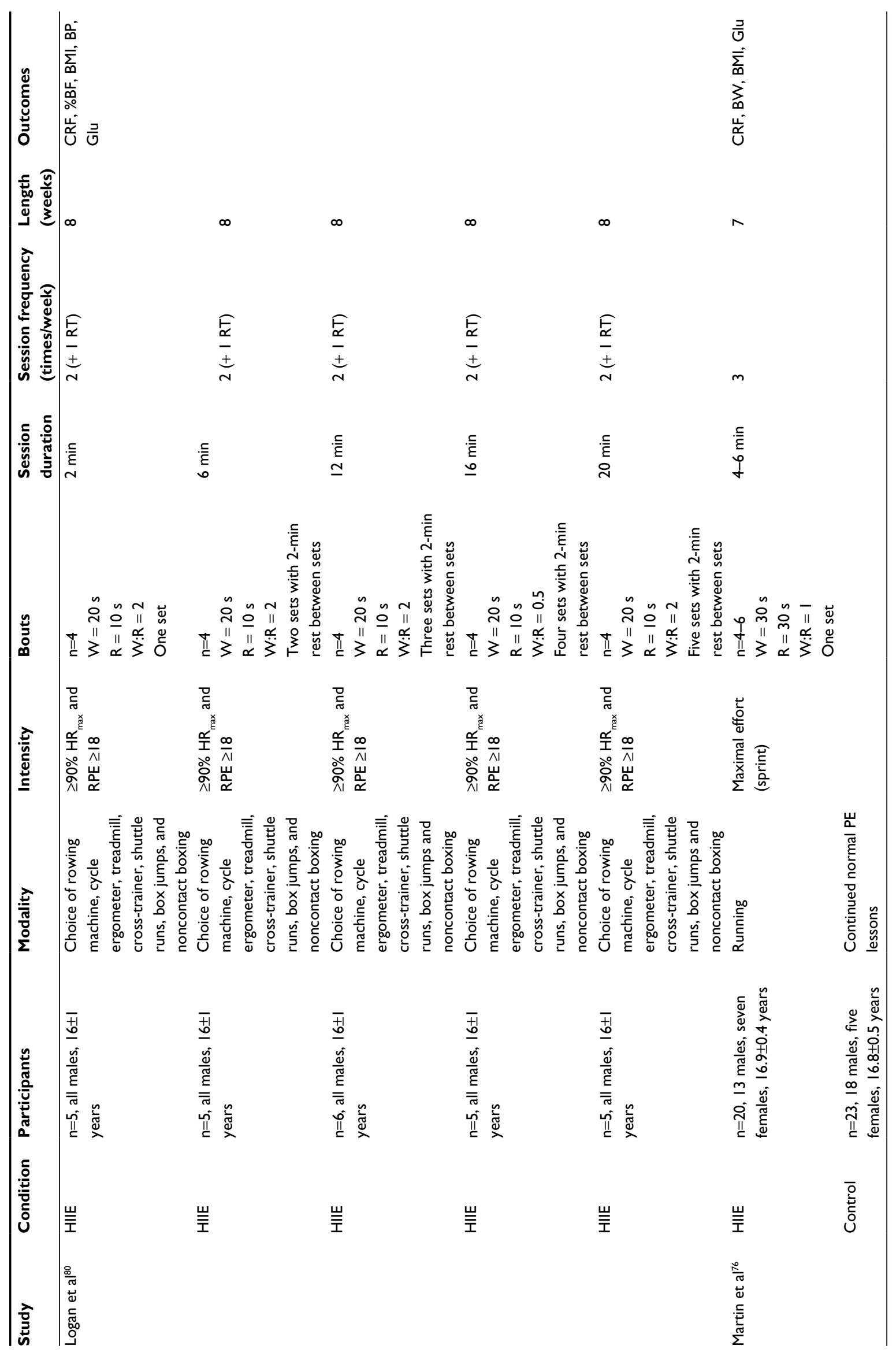




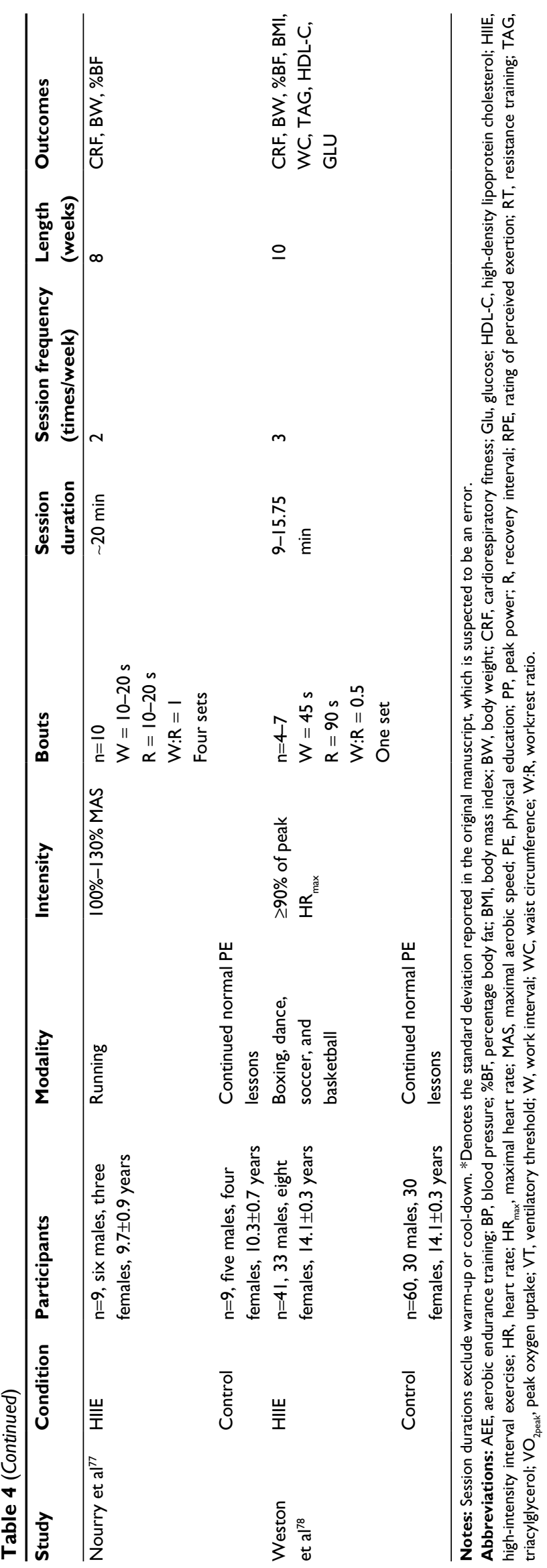

Five ${ }^{66,68,69,75,77}$ nine,${ }^{67,70,72-74,76,78-80}$ and one ${ }^{71}$ took place in primary, secondary, and special education schools, respectively.

\section{Study design}

All but one study ${ }^{72}$ included a control and/or active comparator group. Largely, control groups continued normal physical education (PE) lessons. In studies containing an active comparator, either continuous $\mathrm{AEE}^{68,71,74}$ or a specific HIIE training dose was prescribed. ${ }^{79,80}$ One and two trials, respectively, did not provide information on group assignment procedures ${ }^{67}$ or how randomization occurred. ${ }^{76,77}$ Two utilized a nonrandomized design, ${ }^{66,78}$ whereby group allocation occurred at the school ${ }^{78}$ or class level. ${ }^{66}$ Four $^{70,75,79,80}$ and two ${ }^{68,69}$ trials, respectively, randomized participants individually or by school class (cluster randomization). Boer et $\mathrm{al}^{71}$ and Buchan et $\mathrm{al}^{73,74}$ adopted quasiexperimental designs. Only a quarter of included studies thus utilized the public health and medicine gold standard of an individually randomized controlled trial. ${ }^{81}$ Whether this gold standard is universally suitable for school-based interventions, though, is debatable. Indeed, some previous school-based exercise interventions have opted against individual randomization to mitigate potential contamination effects. ${ }^{82}$ This is an important consideration for school-based HIIE training interventions if, for example, individuals in the same PE class are assigned to different conditions but then exposed to what another treatment entails (eg, watching HIIE training take place). Such a scenario could lead to contamination issues, such as compensatory rivalry (eg, performing activities in addition to and/or at a greater intensity than prescribed) or resentful demoralization (eg, disengagement from the study process) ${ }^{83}$ which may impact on study compliance and/or outcomes influenced by participants' effort (eg, CRF testing). From a practical perspective, cluster randomization may be necessitated by such factors as pupil numbers, abilities, behavior, and schoolteachers' preferences, despite diminishing returns in statistical power and precision. ${ }^{84}$ Therefore, no "one-size-fits-all" design exists for school-based HIIE training interventions.

\section{Participants}

All but two studies ${ }^{70,80}$ recruited a mixture of males and females. Nevertheless, on average only $37 \%$ of participants were girls, highlighting an underrepresentation of females in the pediatric HIIE training literature. Surprisingly few studies acknowledged this imbalance as a potential limitation of their findings, with only two ${ }^{78,79}$ offering possible explanations as to why more girls did not participate. It appeared factors at the 
school level may have hindered recruitment, as participants were recruited from a school containing more boys than girls ${ }^{79}$ or researchers were only able to recruit from one all-male PE class at one intervention school. ${ }^{78}$

The median number of participants per study was 55 (intervention and control combined, interquartile range 26-89). Three studies provided power calculations to justify their sample size. ${ }^{75,76,79}$ Only four, however, provided recruitment rate data (eg, number of pupils targeted vs number providing consent). ${ }^{73,78-80}$ In effectiveness trials, such information is crucial for establishing the reach of an intervention (ie, the number of individuals willing to engage), ${ }^{85}$ especially considering ongoing skepticism over the uptake of HIIE training outside laboratory conditions. ${ }^{86}$ Of those that did provide recruitment information, response rates were $19 \%,{ }^{80} 55 \%,{ }^{78} 86 \%,{ }^{79}$ and $97 \%,{ }^{73}$ although the "type" of pupil targeted differed. In the trial by Logan et al, ${ }^{80}$ pupils were eligible to participate only if they were disengaged from physical activity, PE lessons, or any form of extracurricular sport. This contrasts with those in Buchan et al, ${ }^{73}$ which only included pupils choosing to study PE to examination level. While recruitment was not limited by participant attributes in other trials, ${ }^{78,79}$ there were potential sex imbalances, as described previously. With only four examples available to date, far more evidence is required before claims on the potential reach of school-based HIIE training can be confirmed. As such, in future trials, researchers should provide data on recruitment-response rates.

\section{HIIE training intervention protocols}

The average length of a school-based HIIE training intervention was 7 weeks (range: 2-15 weeks). Despite a lack of specific information, it could be assumed that trials conducted over 7 weeks spanned one school term/semester. Trials lasting longer than one school term (eg, $>7$ weeks $)^{67,71,78}$ may inevitably include school holidays too, which could impact on study outcomes. Given the lack of flexibility in school term dates, the inclusion of holiday periods may be an unavoidable consequence of longer school-based HIIE training trials. Regarding exercise frequency, all but one trial ${ }^{67}$ provided two to three intervention sessions a week. In six trials, ${ }^{66-69,75,77}$ HIIE training or AEE sessions were conducted in addition to regular PE. Interestingly, all but of one of these ${ }^{67}$ were conducted in primary schools. Though speculative, it could be argued that the addition of extra activities to the school timetable may be more feasible at primary level than at secondary level, where competing interests and expectations from other subjects negate flexibility. Six secondary school-based studies embedded their interventions by substituting PE with HIIE training or AEE sessions, ${ }^{71,73,74,76,78,79}$ with two ${ }^{78,79}$ providing an additional session during school lunch breaks ${ }^{79}$ and/or after school ${ }^{78}$ In one trial, all intervention sessions took place during the school lunch break, ${ }^{80}$ and in another, sessions were conducted in morning form time. ${ }^{70}$ From a sustainability perspective, it is encouraging that researchers are looking beyond purely PE lessons for intervention delivery, as it is not the purpose of HIIE training to replace PE or its wider learning objectives in the long term. Nevertheless, the timeefficient nature of HIIE training does lend itself to existing alongside PE. Indeed, it is plausible that HIIE activities that complement the main PE lesson could be done at the start of a class, for example, thus not taking over the entire lesson.

Across the majority of school-based HIIE training interventions, detailed information on exercise modes and session content was provided. This is commendable, as traditionally the quality of intervention reporting, particularly exercise trials, is poor. ${ }^{87,88}$ In school-based HIIE training interventions, however, authors often provided diagrams ${ }^{67,74}$ and tables containing intervention activities ${ }^{66,75,78}$ to aid quantification. Individual HIIE repetitions ranged from 10 to 60 seconds in length with work:rest ratios $\leq 1$, with two exceptions. ${ }^{75,80}$ The number of repetitions performed per HIIE training session ranged from 4 to 40 across studies, with repetitions of the shortest duration (eg, 10 seconds) often performed the largest number of times. Five ${ }^{66-69,77}$ and three studies, ${ }^{73,74,76}$ respectively, utilized a running protocol by Baquet et $\mathrm{al}^{67}$ or Buchan et al. ${ }^{74}$ Two studies utilized cycle ergometry models. ${ }^{71,72}$ The remainder ${ }^{70,75,78-80}$ shunned traditional exercise modes in favor of more contemporary activities. In the work of Lambrick et al, ${ }^{75} \mathrm{HIIE}$ training constituted such games as dodgeball, with Boddy et $\mathrm{al}^{70}$ devising a dance-based HIIE training program. Following focus group consultations with adolescent school pupils, Weston et $\mathrm{al}^{78}$ devised a school-based HIIE training program consisting of boxing, dance, basketball, and soccer drills, with the activity rotated on a weekly basis. In the trial by Logan et al, ${ }^{80}$ HIIE training was conducted on preexisting PE equipment (rowing machines, treadmills, and cross-trainers), thus negating the need for specialized equipment. Similarly, in Costigan et al, ${ }^{79} \mathrm{HIIE}$ training was performed with minimal or no equipment (shuttle runs, skipping, and BW exercises).

Encouragingly, the majority of studies attempted to monitor the intensity of their HIIE training, thus providing an objective measure of the extent to which participants complied with the prescribed exercise dose. ${ }^{89}$ Four and one studies, respectively, prescribed the intensity of HIIE training as a percentage of $\mathrm{MAS}^{67-69,77}$ or ventilatory threshold, ${ }^{71}$ but 
did not confirm whether these were complied with. Two studies collected ratings of perceived exertion. ${ }^{72,80}$ Nine studies $^{66,70,73-76,78-80}$ presented HIIE intervention HR data in either absolute or relative $\left(\% \mathrm{HR}_{\max }\right)$ units. With the exception of one study, however, ${ }^{78}$ only mean and between-subject SD data were reported. As repeated HIIE training sessions performed across an intervention will give rise to betweensubject and within-subject variability in exercise-intensity response, researchers are guided toward using statistical methods that enable the variability to be properly separated. ${ }^{89}$

To quantify school-based HIIE training intervention dose further, several authors also provided information on session attendance and intervention dropout rates. In five trials, failure to attend a specific percentage of sessions and/or missing outcome data led to exclusion from data analysis. ${ }^{68,69,76,77,80}$ Of the seven studies providing these data, ${ }^{70-73,78-80}$ the average retention \pm SD was $91.9 \% \pm 5.8 \%$. Though this figure is encouraging, it represents under half of the 15 included studies. To allow a fuller picture on retention, therefore, all future school-based HIIE training studies should provide explicit retention data. Regarding session attendance, studies provided information in the following formats: percentage of total sessions attended ${ }^{78}$ mean intervention time completed, ${ }^{70}$ mean number of sessions attended, ${ }^{73,74}$ minimum and maximum number of sessions attended, ${ }^{71}$ and average number of sessions attended per week. ${ }^{79}$ Reasons for missing sessions included illness, educational commitments, holidays, forgetfulness, and lack of appropriate clothing. Across all school HIIE training interventions, no training-related injuries or adverse events were reported. In three trials, one to three participants ${ }^{78,80,72}$ did not complete their designated HIIE training due to illness or unrelated injuries. Regarding incentives, one study offered a $£ 5$ gift voucher to all participants ${ }^{70}$ and another awarded "trainer of the day" certificates and gift vouchers to intervention participants during and on completion of the intervention. ${ }^{79}$ In the work of Weston et al, ${ }^{78}$ all participants received a thank you pack and HIIE training intervention participants completing $\geq 70 \%$ and $\geq 90 \%$ of sessions, respectively, received a T-shirt and entered a prize draw to win a pair of running shoes.

\section{Effects of school-based HIIE training on cardiometabolic health and fitness}

Changes in CRF following school-based HIIE training were examined in all 15 studies. Seven and eight studies, respectively, measured CRF directly through gas analysis $\left(\mathrm{eg}, \mathrm{VO}_{2 \text { peak }}\right)$ and a laboratory-based $\mathrm{CPET}^{68,70-}$ $72,75,77,80$ or indirectly via 20 -meter multistage fitness-test performance. ${ }^{66,67,69,73,74,76,78,79}$ Eleven reported clear improvements post-HIIE training (defined herein as effects that were statistically significant and/or a small standardized mean difference [effect size $\geq 0.2$ ]). ${ }^{66-69,71,73-75,77,79,80}$ Regarding body-size measures, no studies detected clear improvements in BW following school-based HIIE training. Of the nine trials including BMI-outcome data, ${ }^{67,68,70,73-76,78-80}$ two reported clear improvements. ${ }^{74,79}$ Closer inspection of the data in one study, ${ }^{74}$ however, revealed the significant effect was likely due to participants growing in stature, rather than a reduction in BW. Ten studies examined the effect of school-based HIIE training on percentage $B F, 66,67,69-71,74-78$ of which three ${ }^{71,74,80}$ reported clear reductions. In one, however, twice-weekly HIIE training was supplemented with a weekly resistancetraining session, ${ }^{80}$ which could also feasibly alter body composition. As determining the isolated effect of HIIE training on percentage BF was not possible, caution is warranted in the interpretation of this finding. With regard to waist circumference, four of six studies ${ }^{71,75,78,79}$ reported clear reductions post-HIIE training, though in one trial ${ }^{75}$ this applied only to obese participants. Of the seven studies reporting changes in $\mathrm{BP},{ }^{70-74,78,80}$ four and one, respectively, reported clear reductions in $\mathrm{SBP}^{71,73,74,80}$ and $\mathrm{DBP}^{80}$ An unexpected clear increase in DBP was also observed in HIIE training intervention participants in one study. ${ }^{70}$ Five studies ${ }^{71-74,78}$ examined changes in HDL-C and TAG following schoolbased HIIE training. One observed clear improvements in HDL-C and TAG ${ }^{71}$ with one further study also reporting clear reductions in TAG compared to control participants. ${ }^{78}$ Lastly, seven studies measured changes in blood glucose following school-based HIIE training, ${ }^{71-74,76,78,80}$ and apart from one unexplained clear increase post-HIIE training intervention, ${ }^{74}$ no clear differences were observed.

\section{Perspectives}

Though still in its relative infancy from a research standpoint, the use of school-based HIIE training as a health promotion tool has considerable scope and is already being explored across different continents, climates, and school systems. Given that 11 of the 15 studies included were conducted in the last decade, interest in school-based HIIE training is likely to increase further. Despite some methodological challenges, it appears school-based HIIE training can positively impact outcomes, such as CRF, some body composition measures, and SBP. More work is needed to establish whether schoolbased HIIE training can improve traditional cardiometabolic risk markers, such as blood lipid and glucose profiles, and more novel risk factors, such as blood vessel function. 
Regarding the design and evaluation of school-based HIIE training programs, researchers may consider gaining the insight of those directly involved in their studies (ie, school pupils and teachers). While process evaluations of school-based HIIE training interventions exist, ${ }^{73,79}$ more in-depth explorations could be useful for researchers, pupils, and teachers. From a design and/or outcome perspective, researchers should be mindful that they could face a trade-off between what is scientifically most important and what is feasible and acceptable at the school level. Nonetheless, school-based HIIE training interventions can be delivered and evaluated with methodological rigor, provided adequate consideration is given to some of issues discussed in this review from the outset. Currently, the impact of school-based HIIE training has been assessed on a relatively small scale by individual research groups and institutions. In the future, therefore, such groups should aim to create cross-university and country collaborations to facilitate the design, implementation, and evaluation of larger schoolbased HIIE training trials across multiple study sites.

\section{Conclusion}

The literature synthesized in this narrative review demonstrates that HIIE exercise confers either similar or superior benefits than a comparable bout of AEE across a wide range of cardiometabolic outcomes in children and adolescents. This was evident when considering an acute bout of HIIE, in the application of HIIE training to promote cardiometabolic health in overweight and obese children and adolescents, and in the school setting. It is also important to highlight that a single bout of HIIE is perceived to be more enjoyable $33-36,39$ than a bout of AEE, due to elevated feelings of reward, excitement, and success, ${ }^{90}$ which may serve as a useful strategy for the promotion of HIIE to improve health outcomes. Accordingly, there is a strong rationale for future work to explore how HIIE may be optimized for cardiometabolic benefit and enjoyment across a range of pediatric groups. From a wider health perspective, the impact of school-based HIIE training on overall physical activity levels, CRF, quality of life, and mental health outcomes should also be explored in more depth, but some early data on both topics are promising. ${ }^{78,91}$

\section{Disclosure}

The authors report no conflicts of interest in this work.

\section{References}

1. Berenson GS, Wattigney WA, Tracy RE, et al. Atherosclerosis of the aorta and coronary arteries and cardiovascular risk factors in persons aged 6 to 30 years and studied at necropsy (the Bogalusa Heart Study). Am J Cardiol. 1992;70(9):851-858.
2. Laitinen TT, Pahkala K, Magnussen CG, et al. Ideal cardiovascular health in childhood and cardiometabolic outcomes in adulthood: the Cardiovascular Risk in Young Finns study. Circulation. 2012;125(16):1971-1978.

3. Janssen I, Leblanc AG. Systematic review of the health benefits of physical activity and fitness in school-aged children and youth. Int $J$ Behav Nutr Phys Act. 2010;7:40.

4. World Health Organization. Global Recommendations on Physical Activity for Health. Geneva: WHO; 2012.

5. Hallal PC, Andersen LB, Bull FC, et al. Global physical activity levels: surveillance progress, pitfalls, and prospects. Lancet. 2012; 380(9838):247-257.

6. Metcalf B, Henley W, Wilkin T. Effectiveness of intervention on physical activity of children: systematic review and meta-analysis of controlled trials with objectively measured outcomes (EarlyBird 54). BMJ. 2012;345:e5888.

7. Borde R, Smith JJ, Sutherland R, Nathan N, Lubans DR. Methodological considerations and impact of school-based interventions on objectively measured physical activity in adolescents: a systematic review and meta-analysis. Obes Rev. 2017;18(4):476-490.

8. Hay J, Maximova K, Durksen A, et al. Physical activity intensity and cardiometabolic risk in youth. Arch Pediatr Adolesc Med. 2012;166(11): 1022-1029.

9. Carson V, Rinaldi RL, Torrance B, et al. Vigorous physical activity and longitudinal associations with cardiometabolic risk factors in youth. Int J Obes (Lond). 2014;38(1):16-21.

10. Ruiz JR, Rizzo NS, Hurtig-Wennlöf A, Ortega FB, Wärnberg J, Sjöström M. Relations of total physical activity and intensity to fitness and fatness in children: the European Youth Heart Study. Am J Clin Nutr. 2006;84(2):299-303.

11. Ortega FB, Ruiz JR, Sjöström M. Physical activity, overweight and central adiposity in Swedish children and adolescents: the European Youth Heart Study. Int J Behav Nutr Phys Act. 2007;4:61.

12. Hopkins ND, Stratton G, Tinken TM, et al. Relationships between measures of fitness, physical activity, body composition and vascular function in children. Atherosclerosis. 2009;204(1):244-249.

13. Aggoun Y, Farpour-Lambert NJ, Marchand LM, Golay E, Maggio $\mathrm{AB}$, Beghetti M. Impaired endothelial and smooth muscle functions and arterial stiffness appear before puberty in obese children and are associated with elevated ambulatory blood pressure. Eur Heart J. 2008;29(6):792-799.

14. Juonala M, Viikari JS, Laitinen T, et al. Interrelations between brachial endothelial function and carotid intima-media thickness in young adults: the Cardiovascular Risk in Young Finns study. Circulation. 2004;110(18):2918-2923.

15. Costigan SA, Eather N, Plotnikoff RC, Taaffe DR, Lubans DR. High-intensity interval training for improving health-related fitness in adolescents: a systematic review and meta-analysis. Br J Sports Med. 2015;49(19):1253-1261.

16. Garcia-Hermoso A, Cerrillo-Urbina AJ, Herrera-Valenzuela T, CristiMontero C, Saavedra JM, Martinez-Vizcaino V. Is high-intensity interval training more effective on improving cardiometabolic risk and aerobic capacity than other forms of exercise in overweight and obese youth? A meta-analysis. Obes Rev. 2016;17(6):531-540.

17. Logan GR, Harris N, Duncan S, Schofield G. A review of adolescent high-intensity interval training. Sports Med. 2014;44(8):1071-1085.

18. Eddolls WT, McNarry MA, Stratton G, Winn CO, Mackintosh KA. High-intensity interval training interventions in children and adolescents: a systematic review. Sports Med. Epub 2017 Jun 22.

19. Kanaley JA, Boileau RA. The onset of the anaerobic threshold at three stages of physical maturity. J Sports Med Phys Fitness. 1988;28(4): 367-374.

20. Dawson EA, Green DJ, Cable NT, Thijssen DH. Effects of acute exercise on flow-mediated dilatation in healthy humans. J Appl Physiol (1985). 2013;115(11):1589-1598.

21. Freese EC, Gist NH, Acitelli RM, et al. Acute and chronic effects of sprint interval exercise on postprandial lipemia in women at-risk for the metabolic syndrome. J Appl Physiol (1985). 2015;118(7):872-879. 
22. Herd SL, Lawrence JE, Malkova D, Murphy MH, Mastana S, Hardman AE. Postprandial lipemia in young men and women of contrasting training status. J Appl Physiol (1985). 2000;89(5):2049-2056.

23. Tsetsonis NV, Hardman AE, Mastana SS. Acute effects of exercise on postprandial lipemia: a comparative study in trained and untrained middle-aged women. Am J Clin Nutr. 1997;65(2):525-533.

24. Sedgwick MJ, Morris JG, Nevill ME, Barrett LA. Effect of repeated sprints on postprandial endothelial function and triacylglycerol concentrations in adolescent boys. J Sports Sci. 2015;33(8):806-816.

25. Chuensiri N, Tanaka H, Suksom D. The acute effects of supramaximal high-intensity intermittent exercise on vascular function in lean vs. obese prepubescent boys. Pediatr Exerc Sci. 2015;27(4):503-509.

26. Morrison JA, Glueck CJ, Horn PS, Yeramaneni S, Wang P. Pediatric triglycerides predict cardiovascular disease events in the fourth to fifth decade of life. Metabolism. 2009;58(9):1277-1284.

27. Barrett LA, Morris JG, Stensel DJ, Nevill ME. Exercise and postprandial plasma triacylglycerol concentrations in healthy adolescent boys. Med Sci Sports Exerc. 2007;39(1):116-122.

28. Thackray AE, Barrett LA, Tolfrey K. Acute high-intensity interval running reduces postprandial lipemia in boys. Med Sci Sports Exerc. 2013;45(7):1277-1284.

29. Tolfrey K, Doggett A, Boyd C, Pinner S, Sharples A, Barrett L. Postprandial triacylglycerol in adolescent boys: a case for moderate exercise. Med Sci Sports Exerc. 2008;40(6):1049-1056.

30. Zhang JQ, Smith B, Langdon MM, et al. Changes in LPLa and reverse cholesterol transport variables during 24 -h postexercise period. Am J Physiol Endocrinol Metab. 2002;283(2):E267-E274.

31. Thackray AE, Barrett LA, Tolfrey K. High-intensity running and energy restriction reduce postprandial lipemia in girls. Med Sci Sports Exerc. 2016;48(3):402-411.

32. Tolfrey K, Thackray AE, Barrett LA. Acute exercise and postprandial lipemia in young people. Pediatr Exerc Sci. 2014;26(2):127-137.

33. Bond B, Gates PE, Jackman SR, Corless LM, Williams CA, Barker AR. Exercise intensity and the protection from postprandial vascular dysfunction in adolescents. Am J Physiol Heart Circ Physiol. 2015;308(11):H1443-H1450.

34. Bond B, Williams CA, Isic C, et al. Exercise intensity and postprandial health outcomes in adolescents. Eur J Appl Physiol. 2015;115(5):927-936.

35. Bond B, Williams CA, Jackman SR, Woodward A, Armstrong N, Barker AR. Accumulating exercise and postprandial health in adolescents. Metabolism. 2015;64(9):1068-1076.

36. Cockcroft EJ, Williams CA, Jackman SR, Bassi S, Armstrong N, Barker AR. A single bout of high-intensity interval exercise and work-matched moderate-intensity exercise has minimal effect on glucose tolerance and insulin sensitivity in 7- to 10-year-old boys. J Sports Sci. Epub 2017 Feb 13.

37. Cockcroft EJ, Williams CA, Tomlinson OW, et al. High intensity interval exercise is an effective alternative to moderate intensity exercise for improving glucose tolerance and insulin sensitivity in adolescent boys. J Sci Med Sport. 2015;18(6):720-724.

38. Riddoch CJ, Mattocks C, Deere K, et al. Objective measurement of levels and patterns of physical activity. Arch Dis Child. 2007;92(11):963-969.

39. Bond B, Hind S, Williams CA, Barker AR. The acute effect of exercise intensity on vascular function in adolescents. Med Sci Sports Exerc. 2015;47(12):2628-2635.

40. Burns SF, Oo HH, Tran AT. Effect of sprint interval exercise on postexercise metabolism and blood pressure in adolescents. Int J Sport Nutr Exerc Metab. 2012;22(1):47-54.

41. Crisp NA, Fournier PA, Licari MK, Braham R, Guelfi KJ. Adding sprints to continuous exercise at the intensity that maximises fat oxidation: implications for acute energy balance and enjoyment. Metabolism. 2012;61(9):1280-1288.

42. Mills A, Rosenberg M, Stratton G, et al. The effect of exergaming on vascular function in children. $J$ Pediatr. 2013;163(3):806-810.

43. $\mathrm{Ng} \mathrm{M}$, Fleming $\mathrm{T}$, Robinson $\mathrm{M}$, et al. Global, regional, and national prevalence of overweight and obesity in children and adults during 1980-2013: a systematic analysis for the Global Burden of Disease Study 2013. Lancet. 2014;384(9945):766-781.
44. Lobstein T, Jackson-Leach R. Estimated burden of paediatric obesity and co-morbidities in Europe - part 2: numbers of children with indicators of obesity-related disease. Int J Pediatr Obes. 2006;1(1):33-41.

45. Ingul CB, Tjonna AE, Stolen TO, Stoylen A, Wisloff U. Impaired cardiac function among obese adolescents: effect of aerobic interval training. Arch Pediatr Adolesc Med. 2010;164(9):852-859.

46. Reilly JJ, Kelly J. Long-term impact of overweight and obesity in childhood and adolescence on morbidity and premature mortality in adulthood: systematic review. Int J Obes (Lond). 2011;35(7):891-898.

47. Kumar S, Kelly AS. Review of Childhood obesity: from epidemiology, etiology, and comorbidities to clinical assessment and treatment. Mayo Clin Proc. 2017;92(2):251-265.

48. Stoner L, Rowlands D, Morrison A, et al. Efficacy of exercise intervention for weight loss in overweight and obese adolescents: meta-analysis and implications. Sports Med. 2016;46(11):1737-1751.

49. Lau PW, Wong DP, Ngo JK, Liang Y, Kim CG, Kim HS. Effects of high-intensity intermittent running exercise in overweight children. Eur J Sport Sci. 2015;15(2):182-190.

50. de Araujo AC, Roschel H, Picanço AR, et al. Similar health benefits of endurance and high-intensity interval training in obese children. PloS One. 2012;7(8):e42747.

51. Kargarfard M, Lam ET, Shariat A, et al. Effects of endurance and high intensity training on ICAM-1 and VCAM-1 levels and arterial pressure in obese and normal weight adolescents. Phys Sportsmed. 2016;44(3):208-216.

52. Murphy A, Kist C, Gier AJ, Edwards NM, Gao Z, Siegel RM. The feasibility of high-intensity interval exercise in obese adolescents. Clin Pediatr (Phila). 2015;54(1):87-90.

53. Starkoff BE, Eneli IU, Bonny AE, Hoffman RP, Devor ST. Estimated aerobic capacity changes in adolescents with obesity following high intensity interval exercise. Int J Kinesiol Sports Sci. 2014;2(3):1-8.

54. Tjonna AE, Stølen TO, Bye A, et al. Aerobic interval training reduces cardiovascular risk factors more than a multitreatment approach in overweight adolescents. Clin Sci (Lond). 2009;116(4):317-326.

55. Koubaa A, Trabelsi H, Masmoudi L, et al. Effect of intermittent and continuous training on body composition cardiorespiratory fitness and lipid profile in obese adolescents. J Pharm. 2013;3(2):31-37.

56. Lazzer S, Tringali G, Caccavale M, De Micheli R, Abbruzzese L, Sartorio A. Effects of high-intensity interval training on physical capacities and substrate oxidation rate in obese adolescents. $J$ Endocrinol Invest. 2017;40(2):217-226.

57. Racil G, Ounis OB, Hammouda O, et al. Effects of high vs. moderate exercise intensity during interval training on lipids and adiponectin levels in obese young females. Eur J Appl Physiol. 2013;113(10):2531-2540.

58. Racil G, Coquart JB, Elmontassar W, et al. Greater effects of highcompared with moderate-intensity interval training on cardio-metabolic variables, blood leptin concentration and ratings of perceived exertion in obese adolescent females. Biol Sport. 2016;33(2):145-152.

59. Cole TJ, Bellizzi MC, Flegal KM, Dietz WH. Establishing a standard definition for child overweight and obesity worldwide: international survey. BMJ. 2000;320(7244):1240-1243.

60. Ortega FB, Ruiz JR, Castillo MJ, Sjöström M. Physical fitness in childhood and adolescence: a powerful marker of health. Int J Obes (Lond). 2008;32(1):1-11.

61. Eisenmann JC, Wickel EE, Welk GJ, Blair SN. Relationship between adolescent fitness and fatness and cardiovascular disease risk factors in adulthood: the Aerobics Center Longitudinal Study (ACLS). Am Heart J. 2005;149(1):46-53.

62. Högström G, Nordström A, Nordström P. Aerobic fitness in late adolescence and the risk of early death: a prospective cohort study of 1.3 million Swedish men. Int J Epidemiol. Epub 2015 Dec 20.

63. Armstrong N, Barker AR. Endurance training and elite young athletes. Medicine Sport Sci. 2011;56:59-83.

64. Kriemler S, Meyer U, Martin E, van Sluijs EM, Andersen LB, Martin BW. Effect of school-based interventions on physical activity and fitness in children and adolescents: a review of reviews and systematic update. Br J Sports Med. 2011;45(11):923-930. 
65. Zimmet P, Alberti KG, Kaufman F, et al. The metabolic syndrome in children and adolescents: an IDF consensus report. Pediatr Diabetes. 2007;8(5):299-306.

66. Baquet G, Berthoin S, Dupont G, Blondel N, Fabre C, van Praagh E. Effects of high intensity intermittent training on peak $\mathrm{VO}_{2}$ in prepubertal children. Int J Sports Med. 2002;23(6):439-444.

67. Baquet G, Berthoin S, Gerbeaux M, van Praagh E. High-intensity aerobic training during a 10 week one-hour physical education cycle: effects on physical fitness of adolescents aged 11 to 16. Int J Sports Med. 2001;22(4):295-300.

68. Baquet G, Gamelin FX, Mucci P, Thévenet D, van Praagh E, Berthoin $\mathrm{S}$. Continuous vs. interval aerobic training in 8- to 11-year-old children. J Strength Cond Res. 2010;24(5):1381-1388.

69. Baquet G, Guinhouya C, Dupont G, Nourry C, Berthoin S. Effects of a short-term interval training program on physical fitness in prepubertal children. J Strength Cond Res. 2004;18(4):708-713.

70. Boddy LM, Stratton G, Hackett AF, George KP. The effectiveness of a "short, sharp, shock" high intensity exercise intervention in 11- and 12-year-old Liverpool schoolgirls. Arch Exerc Health Dis. 2010;1(1): 19-25.

71. Boer PH, Meeus M, Terblanche E, et al. The influence of sprint interval training on body composition, physical and metabolic fitness in adolescents and young adults with intellectual disability: a randomized controlled trial. Clin Rehabil. 2014;28(3):221-231.

72. Bond B, Cockcroft EJ, Williams CA, et al. Two weeks of high-intensity interval training improves novel but not traditional cardiovascular disease risk factors in adolescents. Am J Physiol Heart Circ Physiol. 2015;309(6):H1039-H1047.

73. Buchan DS, Ollis S, Young JD, Cooper SM, Shield JP, Baker JS. High intensity interval running enhances measures of physical fitness but not metabolic measures of cardiovascular disease risk in healthy adolescents. BMC Public Health. 2013;13:498.

74. Buchan DS, Ollis S, Young JD, et al. The effects of time and intensity of exercise on novel and established markers of CVD in adolescent youth. Am J Hum Biol. 2011;23(4):517-526.

75. Lambrick D, Westrupp N, Kaufmann S, Stoner L, Faulkner J. The effectiveness of a high-intensity games intervention on improving indices of health in young children. J Sports Sci. 2016;34(3):190-198.

76. Martin R, Buchan DS, Baker JS, Young J, Sculthorpe N, Grace FM. Sprint interval training (SIT) is an effective method to maintain cardiorespiratory fitness (CRF) and glucose homeostasis in Scottish adolescents. Biol Sport. 2015;32(4):307-313.

77. Nourry C, Deruelle F, Guinhouya C, et al. High-intensity intermittent running training improves pulmonary function and alters exercise breathing pattern in children. Eur J Appl Physiol. 2005;94(4):415-423.
78. Weston KL, Azevedo LB, Bock S, Weston M, George KP, Batterham AM. Effect of novel, school-based high-intensity interval training (HIT) on cardiometabolic health in adolescents - project FFAB (fun fast activity blasts): an exploratory controlled before-and-after trial. PloS One. 2016;11(8):e0159116.

79. Costigan SA, Eather N, Plotnikoff RC, et al. Preliminary efficacy and feasibility of embedding high intensity interval training into the school day: a pilot randomized controlled trial. Prev Med Rep. 2015;2:973-979.

80. Logan GR, Harris N, Duncan S, Plank LD, Merien F, Schofield G. Low-active male adolescents: a dose response to high-intensity interval training. Med Sci Sports Exerc. 2016;48(3):481-490.

81. Murray DM, Varnell SP, Blitstein JL. Design and analysis of grouprandomized trials: a review of recent methodological developments. Am J Public Health. 2004;94(3):423-432.

82. Henaghan J, McWhannell N, Foweather L, et al. The effect of structured exercise classes and a lifestyle intervention on cardiovascular risk factors in primary schoolchildren: an exploratory trial (the A-CLASS project). Pediatr Exerc Sci. 2008;20(2):169-180.

83. Cook TD, Campbell TD. Quasi-Experimentation: Design and Analysis Issues for Field Settings. Boston: Houghton Mifflin; 1979.

84. Hemming K, Eldridge S, Forbes G, Weijer C, Taljaard M. How to design efficient cluster randomised trials. BMJ. 2017;358:j3064.

85. Glasgow RE, Vogt TM, Boles SM. Evaluating the public health impact of health promotion interventions: the RE-AIM framework. Am J Public Health. 1999;89(9):1322-1327.

86. Biddle SJ, Batterham AM. High-intensity interval exercise training for public health: a big HIT or shall we HIT it on the head? Int J Behav Nutr Phys Act. 2015;12:95.

87. Hoffmann TC, Glasziou PP, Boutron I, et al. Better reporting of interventions: template for intervention description and replication (TIDieR) checklist and guide. BMJ. 2014;348:g1687.

88. Mujika I. The alphabet of sport science research starts with Q. Int $J$ Sports Physiol Perform. 2013;8(5):465-466.

89. Taylor KL, Weston M, Batterham AM. Evaluating intervention fidelity: an example from a high-intensity interval training study. PloSOne. 2015;10(4): e0125166.

90. Malik AA, Williams CA, Bond B, Weston KL, Barker AR. Acute cardiorespiratory, perceptual and enjoyment responses to high-intensity interval exercise in adolescents. Eur J Sport Sci. Epub 2017 Sep 2.

91. Costigan SA, Eather N, Plotnikoff RC, Hillman CH, Lubans DR. Highintensity interval training for cognitive and mental health in adolescents. Med Sci Sports Exerc. 2016;48(10):1985-1993.

92. Cederholm J, Wibell L. Insulin release and peripheral sensitivity at the oral glucose tolerance test. Diabetes Res Clin Pract. 1990;10(2):167-175.
Open Access Journal of Sports Medicine

\section{Publish your work in this journal}

The Open Access Journal of Sports Medicine is an international, peer-reviewed, open access journal publishing original research, reports, reviews and commentaries on all areas of sports medicine. The journal is included on PubMed. The manuscript management system is completely online and includes a very quick and fair

\section{Dovepress}

peer-review system. Visit http://www.dovepress.com/testimonials.php to read real quotes from published authors. 